\title{
Efficacy and safety of artemisinin combination therapy (ACT) for non-falciparum malaria: a systematic review
}

Benjamin J Visser ${ }^{1,2,3}$, Rosanne W Wieten ${ }^{1}$, Daniëlle Kroon ${ }^{1,2}$, Ingeborg M Nagel ${ }^{4}$, Sabine Bélard ${ }^{1,5}$, Michèle van Vugt ${ }^{1}$ and Martin P Grobusch ${ }^{1,2,3^{*}}$

\begin{abstract}
Background: Artemisinin combination therapy (ACT) is recommended as first-line treatment for uncomplicated Plasmodium falciparum malaria, whereas chloroquine is still commonly used for the treatment of non-falciparum species (Plasmodium vivax, Plasmodium ovale and Plasmodium malariae). A more simplified, more uniform treatment approach across all malaria species is worthwhile to be considered both in endemic areas and for malaria as an imported condition alike.
\end{abstract}

Methods: A PROSPERO-registered systematic review to determine the efficacy and safety of ACT for the treatment of non-falciparum malaria was conducted, following PRISMA guidelines. Without language restrictions, Medline/ PubMed, Embase, Cochrane Central Register of Controlled Trials, Web of Science, LILACS, Biosis Previews and the African Index Medicus were searched for studies published up to November 2014.

Results: The literature search identified 986 reports; 40 publications were found eligible for inclusion, all of them on non-falciparum malaria in endemic areas. Most evidence was available for $P$. vivax $(n=35)$. Five clinical trials in total were identified evaluating ACT for P. ovale, P. malariae and Plasmodium knowlesi. Most ACT presentations have high efficacy against $P$. vivax parasites; artemisinin-based combinations have shorter parasite and fever clearance times compared to chloroquine. ACT is as effective as chloroquine in preventing recurrent parasitaemia before day 28. Artemisinin-based combinations with long half-lives show significantly fewer recurrent parasitaemia up to day 63. The limited evidence available supports both the use of chloroquine and an ACT for P. ovale and P. malariae. ACT seems to be preferable for optimal treatment of $P$. knowlesi.

Conclusion: $A C T$ is at least equivalent to chloroquine in effectively treating non-falciparum malaria. These findings may facilitate development of simplified protocols for treating all forms of malaria with $A C T$, including returning travellers. Obtaining comprehensive efficacy and safety data on ACT use for non-falciparum species particularly for P. ovale, P. malariae and P. knowlesi should be a research priority.

Trial registration: CRD42014009103

Keywords: Artemisinin combination therapy, ACT, Non-falciparum malaria, Plasmodium vivax, Plasmodium ovale, Plasmodium malariae, Plasmodium knowlesi

\footnotetext{
*Correspondence: m.p.grobusch@amc.uva.nl

${ }^{1}$ Department of Infectious Diseases, Division of Internal Medicine, Center of

Tropical Medicine and Travel Medicine, Academic Medical Center, University

of Amsterdam, Meibergdreef 9, PO Box 22700, 1100 DE Amsterdam, The

Netherlands

${ }^{2}$ Centre de Recherches de Médicales de Lambaréné (CERMEL), Albert

Schweitzer Hospital, Lambaréné, Gabon

Full list of author information is available at the end of the article
} 


\section{Background}

Malaria remains one of the most important and potentially life-threatening parasitic diseases of humans. Five species of the genus Plasmodium are known to cause human malaria infections. The majority of cases are caused by Plasmodium falciparum, which can lead to high morbidity and mortality if not treated promptly. Nonfalciparum malaria is caused by Plasmodium vivax, Plasmodium ovale subspecies, Plasmodium malariae or Plasmodium knowlesi. Data on the epidemiological patterns of the non-falciparum malarias is scarce. In Asia and the Americas, where malaria transmission is generally low and seasonal, P. falciparum and P. vivax malaria have nearly the same prevalence. Plasmodium malariae and $P$. ovale are mainly found in sub-Saharan Africa and comprise $<10 \%$ of isolates. Currently, non-falciparum malaria constitutes approximately $25 \%$ of all annually imported 11,000 cases of malaria in Europe [1-3]. Artemisinin combination therapy $(\mathrm{ACT})$ is recommended as first-line treatment for uncomplicated falciparum malaria [4]. It is considered for traveller's malaria and P. knowlesi in endemic areas, whereas chloroquine (CQ) is still the standard drug for $P$. vivax, $P$. malariae and $P$. ovale in most countries [5].

The different treatment recommendations for $P$. falciparum and non-falciparum malaria may have several drawbacks in clinical practice. Most importantly, misclassification of Plasmodium spp. is common especially where non-falciparum infections are involved, due to lack of training in microscopy and variations in morphological characteristics within and between Plasmodium spp. [6,7]. CQ treatment of misclassified P. falciparum can have severe or even fatal consequences due to widespread CQ resistance of $P$. falciparum. Despite internal and external quality assessments for parasitological diagnosis, discrepant results between microscopy and polymerase chain reaction (PCR) can still occur even in experienced laboratories [8]. Besides, mixed species infections are common [9] and microscopic diagnosis of mixed-species infections is particularly cumbersome $[10,11]$. Rapid diagnostic tests (such as the $\mathrm{NOW}^{\circ}$ ICT MALARIA P.f/P.v. test based on P. falciparum HRP2/ aldolase), are not capable to differentiate safely single species $P$. falciparum infection from concurrent $P$. malariae or $P$. ovale [12]. Those tests fail probably because of the small quantities of antigen circulating due to a low parasitaemia in $P$. malariae and $P$. ovale. An evaluation of three rapid diagnostic test (OptiMAL-IT, BinaxNOW ${ }^{\bullet}$ Malaria and Paramax-3) for the detection of $P$. knowlesi infection demonstrated a low sensitivity (OptiMAL-IT 71\%, BinaxNOW ${ }^{\circ} 29 \%$ and Paramax-3 RDT $40 \%$ for fresh $P$. knowlesi samples) and low specificity and a risk of misdiagnosis of $P$. falciparum or vivax [13]. Although PCR can unambiguously differentiate between subspecies, it is not used in routine clinical care.
Secondly, $P$. vivax resistance to $\mathrm{CQ}$ is emerging in parts of sub-Saharan Africa and South East Asia [14]. A recent meta-analysis to establish the global extent of chloroquine resistance showed chloroquine resistance in $58(53 \%)$ of 113 assessable study sites, spread across most countries that are endemic for P. vivax [15]. The World Health Organization (WHO) recommends the use of ACT also for the treatment of $P$. vivax in affected areas [16]. So far, resistance of $P$. ovale and $P$. malariae to $C Q$ has been reported only once from Indonesia [17].

Finally, intake of $C Q$ is unpopular in many African descendants due to frequently experienced side effects, e.g. pruritus [8] due to CQ's affinity to melanocytes [18]. Artemisinin derivatives are generally well tolerated, and the safety profile of ACT may be by-and-large determined by the partner drug [19]. The clinical impact of non-falciparum malaria infection seems to be more important as it was considered the last three decades. A systematic review and meta-analysis showed $P$. vivax as a major cause of severe malaria and a comparable incidence of severe malaria between $P$. vivax and P. falciparum was found in infants, children and adults [20].

Hence, there might be a compelling rationale to treat all (including returning travellers) diagnosed with malaria with an ACT irrespective of malaria species [21]. More or less identical first-line treatments based on ACT for all malaria species would not only simplify treatment schemes and avoid ineffective CQ treatment of unrecognized $P$. falciparum infection, but also offer logistical benefits in terms of pharmacological management, whilst the need to eliminate P.vivax/P.ovale hypnozoites outside endemic areas has to be accounted for. Moreover, using a combination treatment will less likely result in artemisinin resistance development. However, certainty about the efficacy and safety of ACT for non-falciparum malaria and mixed malaria infections is needed before translating ACT treatment for all malaria infections into practice. This review aims at describing the efficacy and safety of ACT for nonfalciparum malaria.

The overall objective of the review was to summarize available data on efficacy and safety of artemisinin combination therapy (ACT) for the treatment of nonfalciparum malaria. Primary endpoint was to describe the cure rate at day 28; secondary endpoints were to describe cure rates at days $0-3,7,14,21,28,42,56$ and 63 , fever clearance time (FCT), parasite clearance time (PCT) and adverse events profiles [22].

\section{Methods}

This review was conducted in June 2014. The last search was conducted on 16 November 2014. Objectives and inclusion criteria were specified in advance and 
documented in a protocol. Recommendations made by the Preferred Reporting Items for Systematic Reviews and Meta-Analyses (PRISMA) group were followed [23]. This review was registered in advance in PROSPERO (International prospective register of systematic reviews). Registration number: CRD42014009103 [24]. The full methods section is described in Additional file 1 . For the search strategy and selection criteria, see Additional file 2.

\section{Results}

The initial search yielded 2,022 records of which 986 remained after removal of duplicates (see PRISMA flow diagram, Figure 1). Forty records met the inclusion criteria. Of these, $\mathrm{n}=35$ for $P$. vivax malaria and $\mathrm{n}=5$ altogether for $P$. malariae, $P$. ovale or $P$. knowlesi contained the necessary data for the qualitative synthesis. The full text was retrievable for all included records except two $[25,26]$. No study for the treatment of travellers with non-falciparum malaria with ACT in nonendemic high-income countries was identified.

\section{Treatment of Plasmodium vivax malaria}

The epidemiology, clinical presentation and diagnosis of P. vivax are not in the scope of this review and have been covered elsewhere [27].

\section{ACT versus $C Q$}

Fifteen randomized clinical trials (Table 1) compared ACT with CQ monotherapy (with or without primaquine (PQ); the studied artemisinin-based combinations were artemether-lumefantrine (AL) [28-32], dihydroartemisinin-piperaquine (DHA + PP) [33-35], artesunate plus sulphadoxine-pyrimethamine (AS + SP) [36-38], artesunate-pyronaridine (AS + PY) [39], artemi sinin-naphtoquine (AN) [40], or artesunate (AS) [41]

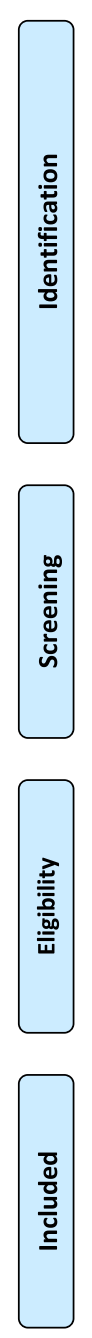

Records identified through database searching (Nov. 2014) $(n=2022)$

Ovid MEDLINE (417), Ovid Embase (673), CENTRAL (72), Web of Science (448), CINAHL (64), African Index Medicus (11), African Journals Online (2), Google Scholar (51), LILACS (6), BIOSIS Previews (229)

Additional records identified through other sources $(n=4)$
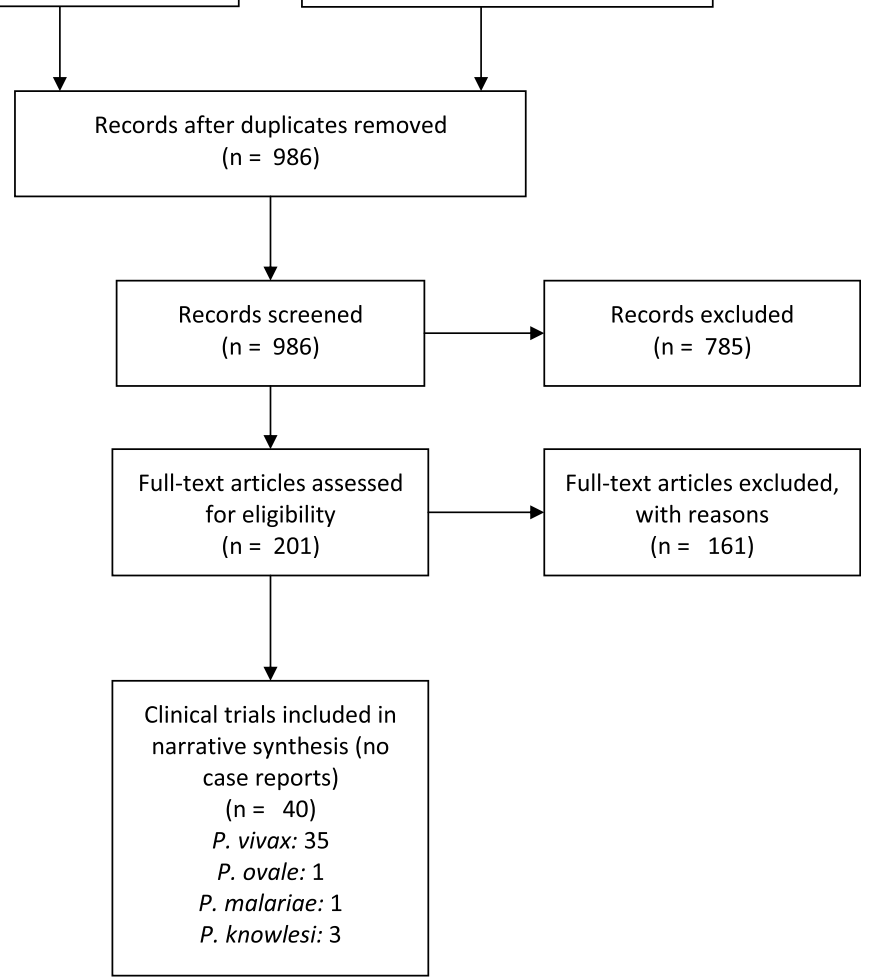

Figure 1 PRISMA flow diagram. From: Moher D, Liberati A, Tetzlaff J, Altman DG, The PRISMA Group (2009). Preferred Reporting Items for Systematic Reviews and Meta-Analyses: The PRISMA Statement. PLoS Med 6: e1000097. 
Table 1 Clinical trials of varying design that specifically report on the effectiveness of an artemisinin derivate combined with a blood schizonticide for the treatment of Plasmodium vivax, P. malariae, $P$. ovale or $P$. knowlesi malaria

\begin{tabular}{|c|c|c|c|c|c|c|c|c|c|c|c|c|c|c|c|c|}
\hline \multirow{2}{*}{$\begin{array}{l}\text { First author, } \\
\text { year of } \\
\text { publication }\end{array}$} & \multirow[t]{2}{*}{ Country } & \multirow[t]{2}{*}{ Study design } & \multirow[t]{2}{*}{ Drug (days) } & \multirow[t]{2}{*}{$\mathrm{N}$} & \multirow{2}{*}{$\begin{array}{l}\text { PCT } \\
\text { (h) }\end{array}$} & \multirow{2}{*}{$\begin{array}{l}\text { FCT } \\
\text { (h) }\end{array}$} & \multicolumn{9}{|c|}{ Cure rate } & \multirow{2}{*}{$\begin{array}{c}\text { Statistical } \\
\text { significance }\end{array}$} \\
\hline & & & & & & & $\begin{array}{c}\text { Day } \\
1\end{array}$ & $\begin{array}{c}\text { Day } \\
2\end{array}$ & $\begin{array}{c}\text { Day } \\
3\end{array}$ & $\begin{array}{c}\text { Day } \\
7\end{array}$ & $\begin{array}{c}\text { Day } \\
14\end{array}$ & $\begin{array}{c}\text { Day } \\
28\end{array}$ & Day 42 & $\begin{array}{c}\text { Day } \\
56\end{array}$ & $\begin{array}{c}\text { Day } \\
63\end{array}$ & \\
\hline \multicolumn{17}{|c|}{ Plasmodium vivax } \\
\hline \multirow[t]{2}{*}{$\begin{array}{l}\text { Lon et al. } 2014 \\
{[50]^{a}}\end{array}$} & Cambodia & $\begin{array}{l}\text { RCT, open } \\
\text { label }\end{array}$ & $\mathrm{DHA}+\mathrm{PP}(2)$ & 32 & $8 \mathrm{~h}$ & $16 \mathrm{~h}$ & NR & NR & NR & NR & NR & NR & $91 \%$ & NR & NR & Day 42: $P=0.62$ \\
\hline & & & $\mathrm{DHA}+\mathrm{PP}(3)$ & 28 & $8 \mathrm{~h}$ & $16 \mathrm{~h}$ & NR & $N R$ & NR & NR & NR & NR & $96 \%$ & NR & NR & \\
\hline \multirow[t]{2}{*}{$\begin{array}{l}\text { Liu et al.2013 } \\
{[44]^{\alpha}}\end{array}$} & China & $\begin{array}{l}\text { RCT, open } \\
\text { label }\end{array}$ & AN (3) & 127 & $26.0 \mathrm{~h}$ & $26.9 \mathrm{~h}$ & $80.3 \%$ & $80.3 \%$ & $100 \%$ & NR & NR & $100 \%$ & $98.4 \%$ & NR & NR & Day 42: $p=0.4496$ \\
\hline & & & $C Q+P Q(3+8)$ & 128 & $37.4 \mathrm{~h}$ & $39.8 \mathrm{~h}$ & $40.6 \%$ & $54.7 \%$ & $100 \%$ & NR & NR & $100 \%$ & $96.1 \%$ & NR & NR & \\
\hline \multirow{2}{*}{$\begin{array}{l}\text { Leang et al. } 2013 \\
{[33]^{\beta}}\end{array}$} & Cambodia & $\mathrm{CCT}$ & $\mathrm{DHA}+\mathrm{PP}$ & 168 & NR & NR & $85.5 \%$ & $100 \%$ & $100 \%$ & NR & NR & $100 \%$ & NR & NR & NR & NR \\
\hline & & & CQ & 211 & NR & NR & $18.9 \%$ & $83.9 \%$ & $98.2 \%$ & NR & NR & $90.5 \%$ & NR & NR & NR & \\
\hline \multirow[t]{2}{*}{$\begin{array}{l}\text { Pasaribu et al. } \\
2013[51]^{a}\end{array}$} & Indonesia & $\begin{array}{l}\mathrm{RCT} \text {, open } \\
\text { label }\end{array}$ & $\begin{array}{c}\mathrm{AAQ}+\mathrm{PQ} \\
(3+14)\end{array}$ & 167 & $<48 \mathrm{~h}$ & NR & NR & $100 \%$ & NR & NR & NR & NR & $91 \%$ & NR & NR & Day 42: $p=0.51$ \\
\hline & & & $\begin{array}{c}\mathrm{DHA}+\mathrm{PP}+\mathrm{PQ} \\
(3+14)\end{array}$ & 164 & $<48 \mathrm{~h}$ & NR & NR & $98.1 \%$ & NR & NR & NR & NR & $94 \%$ & NR & NR & \\
\hline \multirow[t]{3}{*}{$\begin{array}{l}\text { Sutanto et al. } \\
2013[52]^{\alpha}\end{array}$} & Indonesia & $\begin{array}{l}\text { RCT, open } \\
\text { label }\end{array}$ & AS (6) & 41 & $<72 \mathrm{~h}$ & NR & NR & NR & $100 \%$ & $100 \%$ & $100 \%$ & $39,0 \%$ & NR & NR & NR & NR \\
\hline & & & $\begin{array}{c}\mathrm{DHA}+\mathrm{PP}+\mathrm{PQ} \\
(3+14)\end{array}$ & 39 & $<72 \mathrm{~h}$ & NR & NR & NR & $94.9 \%$ & $100 \%$ & $100 \%$ & $100 \%$ & NR & NR & NR & \\
\hline & & & $\mathrm{QN}+\mathrm{PQ}(7+14)$ & 36 & $<72 \mathrm{~h}$ & NR & NR & NR & $97.2 \%$ & $100 \%$ & $100 \%$ & $100 \%$ & NR & NR & NR & \\
\hline \multirow[t]{2}{*}{$\begin{array}{l}\text { Hwang et al. } \\
2013[28]^{\alpha}\end{array}$} & Ethiopia & $\begin{array}{l}\text { RCT, open } \\
\text { label }\end{array}$ & AL (3) & 114 & NR & NR & NR & $100 \%$ & $100 \%$ & NR & NR & $91.1 \%$ & $58.8 \%$ & NR & NR & Day 2: $p=0.006$ \\
\hline & & & CQ (3) & 107 & NR & NR & NR & $94 \%$ & $99,1 \%$ & NR & NR & $97,2 \%$ & $68.4 \%$ & NR & NR & Day 28: $p=0.003$ \\
\hline $\begin{array}{l}\text { Senn et al. } 2013 \\
{[45]^{\alpha}}\end{array}$ & Papua New Guinea & CT & $\mathrm{AL}(\mathrm{NR})$ & 594 & NR & NR & NR & NR & NR & $99.8 \%$ & NR & $97.8 \%$ & $88 \%$ & NR & NR & NR \\
\hline $\begin{array}{l}\text { Mohapatra et al. } \\
2013 \text { [41] NR }\end{array}$ & India & CT & AS (7) & 60 & $48.2 \mathrm{~h}$ & $47.8 \mathrm{~h}$ & NR & NR & NR & NR & NR & $96.6 \%$ & NR & NR & NR & NR \\
\hline $\begin{array}{l}\text { Barber et al. } \\
2013 \text { [46] NA }\end{array}$ & Malaysian Borneo & CT & $\mathrm{AL}+\mathrm{PQ}(+\mathrm{AS})$ & 43 & $48 \mathrm{~h}$ & $24 \mathrm{~h}$ & NR & NR & NR & NR & NR & NR & NR & NR & NR & NR \\
\hline $\begin{array}{l}\text { Abdallah et al. } \\
2012[47]^{a}\end{array}$ & Sudan & CT & $A L+P Q(3+14)$ & 38 & NR & NR & $94.7 \%$ & $100 \%$ & NR & NR & NR & $100 \%$ & NR & NR & NR & NR \\
\hline $\begin{array}{l}\text { Eibach et al. } \\
2012[32]^{\beta}\end{array}$ & Guyana & CT & $\mathrm{AL}+\mathrm{PQ}(3+14)$ & 61 & NR & NR & $100 \%$ & NR & NR & NR & $100 \%$ & $97 \%$ & NR & NR & NR & NR \\
\hline
\end{tabular}


Table 1 Clinical trials of varying design that specifically report on the effectiveness of an artemisinin derivate combined with a blood schizonticide for the treatment of Plasmodium vivax, P. malariae, P. ovale or P. knowlesi malaria (Continued)

\begin{tabular}{|c|c|c|c|c|c|c|c|c|c|c|c|c|c|c|c|c|}
\hline \multirow{4}{*}{$\begin{array}{l}\text { Phyo et al. } 2011 \\
{[34]^{a}}\end{array}$} & Thailand & RCT & $\mathrm{DHA}+\mathrm{PP}(3)$ & 248 & $N R$ & $N R$ & $63,7 \%$ & $97,5 \%$ & $100 \%$ & $N R$ & $N R$ & $95,9 \%$ & $N R$ & $N R$ & $45.1 \%$ & Day 1: $p<0.001$ \\
\hline & & & $\mathrm{CQ}(3)$ & 244 & NR & NR & $21,1 \%$ & $84,1 \%$ & $97.9 \%$ & $N R$ & $N R$ & $90.2 \%$ & NR & NR & $20.9 \%$ & Day 2: $p<0.001$ \\
\hline & & & & & & & & & & & & & & & & Day 28: $p=0.032$ \\
\hline & & & & & & & & & & & & & & & & Day 63: $p<0.001$ \\
\hline \multirow[t]{5}{*}{$\begin{array}{l}\text { Poravuth et al. } \\
2011[39]^{\beta}\end{array}$} & $\begin{array}{l}\text { Cambodia, Thailand, } \\
\text { India and Indonesia }\end{array}$ & RCT, Phase III & $A S+P Y(3)+P Q$ & 228 & $23.0 \mathrm{~h}$ & $15,9 \mathrm{~h}$ & $N R$ & $N R$ & $N R$ & NR & $99.5 \%$ & NR & $N R$ & $N R$ & NR & PCT: $p<0.0001$ \\
\hline & & & & & & & & & & & & & & & & $F C T: p=0.0017$ \\
\hline & & & CQ (3) & 228 & $32.0 \mathrm{~h}$ & $23,8 \mathrm{~h}$ & $N R$ & $N R$ & NR & $N R$ & $100 \%$ & $N R$ & $N R$ & $N R$ & NR & \\
\hline & & & & & & & & & & & & & & & & Difference $(95 \% \mathrm{Cl})$ \\
\hline & & & & & & & & & & & & & & & & Day 14: $-0.5(-2,6-1,4)$ \\
\hline \multirow{2}{*}{$\begin{array}{l}\text { Yohannes et al. } \\
2011 \text { [29] }^{a}\end{array}$} & Ethiopia & ССТ & AL (3) & 88 & $N R$ & $N R$ & $N R$ & $N R$ & $N R$ & $N R$ & $N R$ & $81.0 \%$ & $N R$ & NR & $N R$ & Day 28: $p=0.0145$ \\
\hline & & & CQ (3) & 71 & $N R$ & $N R$ & $N R$ & $N R$ & NR & $N R$ & $N R$ & $92.5 \%$ & $N R$ & $N R$ & $N R$ & \\
\hline \multirow[t]{3}{*}{$\begin{array}{l}\text { Awab et al. } 2010 \\
{[35]^{a}}\end{array}$} & Afghanistan & $\begin{array}{l}\text { RCT, open } \\
\text { label }\end{array}$ & $\mathrm{DHA}+\mathrm{PP}(3)$ & 264 & $N R$ & $N R$ & $91.3 \%$ & $97.8 \%$ & $N R$ & $N R$ & $N R$ & $100 \%$ & $N R$ & $97.2 \%$ & $N R$ & Day 1: $p<0.001$ \\
\hline & & & CQ (3) & 266 & $N R$ & $N R$ & $78.9 \%$ & $97.0 \%$ & $99,2 \%$ & $N R$ & $N R$ & $100 \%$ & $N R$ & $91.1 \%$ & $N R$ & Day $2: p=0.59$ \\
\hline & & & & & & & & & & & & & & & & Day $56: p=0.003$ \\
\hline \multirow[t]{2}{*}{$\begin{array}{l}\text { PiDan et al. } 2009 \\
\text { [25] (abstract only) }\end{array}$} & China & $\mathrm{CT}$ & AS (5) & 30 & $16.3 \mathrm{~h}$ & $38.3 \mathrm{~h}$ & $N R$ & $N R$ & NR & NR & $N R$ & $N R$ & $N R$ & $N R$ & $N R$ & $\begin{array}{c}\text { Cure rate: } 53,3 \% \text { (AS) vs. } \\
56.7 \% \text { (AH). Day: NR }\end{array}$ \\
\hline & & & $\mathrm{AH}(3)$ & 30 & $23.8 \mathrm{~h}$ & $38.5 \mathrm{~h}$ & & & & & & & & & & \\
\hline \multirow[t]{6}{*}{$\begin{array}{l}\text { Krudsood et al. } \\
2008[53]^{\beta}\end{array}$} & Thailand & RCT & $\begin{array}{c}(1) A S+P Q \\
(5+5)\end{array}$ & 68 & $34.9 \mathrm{~h}$ & $16.8 \mathrm{~h}$ & $N R$ & $N R$ & NR & NR & $N R$ & $85 \%$ & $N R$ & $N R$ & $N R$ & $\begin{array}{c}\text { Group } 1 \neq \text { group } 4 \text { and } \\
\text { group } 5(p=0.004 \text { and } \\
0.003) \text {. Group } 2 \neq \text { group } \\
4 \text { and group } 5(p=0.015 \\
\text { and } 0.014)\end{array}$ \\
\hline & & & $\begin{array}{c}\text { (2) } \mathrm{AS}+\mathrm{PQ} \\
(5+7)\end{array}$ & 69 & $36.0 \mathrm{~h}$ & $20.0 \mathrm{~h}$ & $N R$ & $N R$ & NR & NR & $N R$ & $89 \%$ & NR & $N R$ & $N R$ & \\
\hline & & & $\begin{array}{c}\text { (3) } \mathrm{AS}+\mathrm{PQ} \\
(5+9)\end{array}$ & 66 & $35.7 \mathrm{~h}$ & $14.5 \mathrm{~h}$ & $N R$ & $N R$ & NR & NR & $N R$ & $96 \%$ & NR & $N R$ & $N R$ & \\
\hline & & & $\begin{array}{l}\text { (4) } \mathrm{AS}+\mathrm{PQ} \\
(5+11)\end{array}$ & 64 & $34.2 \mathrm{~h}$ & $13.8 \mathrm{~h}$ & $N R$ & $N R$ & $N R$ & NR & $N R$ & $100 \%$ & NR & $N R$ & $N R$ & \\
\hline & & & $\begin{array}{l}\text { (5) } \mathrm{AS}+\mathrm{PQ} \\
(5+14)\end{array}$ & 66 & $37.2 \mathrm{~h}$ & $19.6 \mathrm{~h}$ & NR & $N R$ & NR & NR & $N R$ & $100 \%$ & NR & $N R$ & $N R$ & \\
\hline & & & $\begin{array}{l}\text { (6) } \mathrm{AS}+\mathrm{PQ}(5+7) \\
\text { double dose PQ }\end{array}$ & 66 & $36.8 \mathrm{~h}$ & $21.4 \mathrm{~h}$ & $N R$ & $N R$ & NR & NR & $N R$ & $96 \%$ & NR & $N R$ & $N R$ & \\
\hline
\end{tabular}


Table 1 Clinical trials of varying design that specifically report on the effectiveness of an artemisinin derivate combined with a blood schizonticide for the treatment of Plasmodium vivax, P. malariae, P. ovale or P. knowlesi malaria (Continued)

\begin{tabular}{|c|c|c|c|c|c|c|c|c|c|c|c|c|c|c|c|c|}
\hline \multirow[t]{5}{*}{$\begin{array}{l}\text { Karunajeewa } \\
\text { et al. } 2008[38]^{\beta}\end{array}$} & Papua New Guinea & RCT & $\mathrm{AL}(3)$ & 39 & $1.4 d$ & $2.1 d$ & NR & NR & NR & NR & $N R$ & $55.5 \%$ & $45.5 \%$ & NR & NR & PCT: ACTs vs. \\
\hline & & & & & & & & & & & & & & & & $C Q+S P: p=0.05$ \\
\hline & & & $A S+S P(3)$ & 51 & $1.1 d$ & $2.1 d$ & $N R$ & $N R$ & NR & $N R$ & $N R$ & $63.8 \%$ & $51.3 \%$ & $N R$ & NR & $F C T: p>0.05$ \\
\hline & & & $\mathrm{DHA}+\mathrm{PP}(3)$ & 44 & $1.2 \mathrm{~d}$ & $1.9 \mathrm{~d}$ & NR & $N R$ & NR & $N R$ & NR & $84.2 \%$ & $72.2 \%$ & $N R$ & NR & \\
\hline & & & $C Q+S P(3)$ & 61 & $3.1 \mathrm{~d}$ & $2.3 d$ & NR & $N R$ & NR & NR & $N R$ & $58.8 \%$ & $44.7 \%$ & NR & NR & \\
\hline $\begin{array}{l}\text { Dao et al. } 2007 \\
{[42]^{\beta}}\end{array}$ & Vietnam & $\mathrm{CT}$ & $\mathrm{AS}+\mathrm{PQ}(2+7)$ & 28 & $14.2 \mathrm{~h}$ & $18.6 \mathrm{~h}$ & $N R$ & $N R$ & NR & $N R$ & $N R$ & $96.2 \%$ & $N R$ & $N R$ & NR & $N R$ \\
\hline \multirow[t]{2}{*}{$\begin{array}{l}\text { Hasugian et al. } 2007 \\
{[54]^{a}}\end{array}$} & Papua, Indonesia & RCT & $\begin{array}{c}\mathrm{AAQ}+\mathrm{PQ} \\
(3+14)\end{array}$ & 75 & $<48 \mathrm{~h}$ & $<48 \mathrm{~h}$ & $N R$ & $N R$ & NR & $N R$ & $N R$ & $N R$ & $52 \%$ & $N R$ & NR & Day 42: $p<0.001$ \\
\hline & & & $\begin{array}{c}\mathrm{DHA}+\mathrm{PP}+\mathrm{PQ} \\
(3+14)\end{array}$ & 74 & $<48 \mathrm{~h}$ & $<48 \mathrm{~h}$ & $N R$ & $N R$ & NR & $N R$ & $N R$ & $N R$ & $84 \%$ & $N R$ & NR & \\
\hline \multirow{2}{*}{$\begin{array}{l}\text { Ratcliff et al. } \\
2007[55]^{\alpha}\end{array}$} & Papua, Indonesia & RCT & $\mathrm{AL}(3)$ & 141 & $N R$ & NR & $N R$ & $N R$ & $N R$ & $N R$ & $N R$ & $N R$ & $43 \%$ & $N R$ & NR & $p<0.0001$ \\
\hline & & & $\mathrm{DHA}+\mathrm{PP}(3)$ & 147 & $N R$ & $N R$ & $N R$ & $N R$ & NR & $N R$ & $N R$ & $N R$ & $86 \%$ & $N R$ & NR & \\
\hline \multirow{3}{*}{$\begin{array}{l}\text { Krudsood et al. } \\
2007[31]^{a}\end{array}$} & Thailand & RCT & $A L+P Q(3+14)$ & 47 & $41.6 \mathrm{~h}$ & $21.8 \mathrm{~h}$ & $N R$ & $N R$ & NR & $N R$ & $N R$ & $97.4 \%$ & $N R$ & $N R$ & NR & $P C T: p<0.001$ \\
\hline & & & & & & & & & & & & & & & & $F C T: p=0.12$ \\
\hline & & & $C Q+P Q(3+14)$ & 51 & $55.8 \mathrm{~h}$ & $25.3 \mathrm{~h}$ & $N R$ & $N R$ & $N R$ & NR & $N R$ & $100 \%$ & $N R$ & NR & NR & \\
\hline \multirow{4}{*}{$\begin{array}{l}\text { Kolaczinski et al. } \\
2007[36]^{a}\end{array}$} & Afghanistan & RCT & $\mathrm{AS}+\mathrm{SP}(3+1)$ & 94 & $N R$ & $N R$ & $94.4 \%$ & $100 \%$ & $100 \%$ & $100 \%$ & $100 \%$ & $99 \%$ & $76 \%$ & $N R$ & NR & Difference (95\% Cl) \\
\hline & & & & & & & & & & & & & & & & Day 28: 3.3\% (-2.3-9.8) \\
\hline & & & CQ (3) & 96 & $N R$ & $N R$ & $72.2 \%$ & $97.8 \%$ & $100 \%$ & $100 \%$ & $100 \%$ & $96 \%$ & $54 \%$ & $N R$ & NR & \\
\hline & & & & & & & & & & & & & & & & Day 42: $21.1 \%(7.2-34.0)$ \\
\hline $\begin{array}{l}\text { Yoda et al. } 2005 \\
\text { [26] (abstract only) }\end{array}$ & Thailand & $\mathrm{CT}$ & AS (5) + PQ (14) & 42 & $36.7 \mathrm{~h}$ & $14.6 \mathrm{~h}$ & $N R$ & $N R$ & $N R$ & $N R$ & $N R$ & $95.2 \%$ & $N R$ & $N R$ & NR & NR \\
\hline $\begin{array}{l}\text { Hamedi et al. } \\
2004[48]^{a}\end{array}$ & Thailand & $\mathrm{CT}$ & $A S+P Q(5+14)$ & 42 & $36.7 \mathrm{~h}$ & $14.6 \mathrm{~h}$ & $N R$ & $N R$ & NR & $N R$ & $100 \%$ & $95.2 \%$ & $N R$ & NR & NR & NR \\
\hline $\begin{array}{l}\text { Karunajeewa } \\
\text { et al. } 2003[49]^{a}\end{array}$ & Papua New Guinea & $\mathrm{CT}$ & AS (1) & 5 & $N R$ & NR & $80 \%$ & $N R$ & NR & $N R$ & $N R$ & $N R$ & $N R$ & $N R$ & NR & NR \\
\hline \multirow[t]{4}{*}{$\begin{array}{l}\text { Silachamroon } \\
\text { et al. } 2003[56]^{a}\end{array}$} & Thailand & RCT & AS (5) & 157 & $39.3 \mathrm{~h}$ & $21.2 \mathrm{~h}$ & $N R$ & $N R$ & $N R$ & $N R$ & $N R$ & $47.8 \%$ & $N R$ & $N R$ & NR & $\begin{array}{l}\text { Day 28: } p<0.0001 \text {. AS + } \\
P Q \text { (both) vs. AS(both) }\end{array}$ \\
\hline & & & AS (7) & 159 & $39.6 \mathrm{~h}$ & $21.7 \mathrm{~h}$ & $N R$ & $N R$ & $N R$ & $N R$ & $N R$ & $52.2 \%$ & $N R$ & $N R$ & NR & \\
\hline & & & $\mathrm{AS}+\mathrm{PQ}(5+14)$ & 142 & $37.7 \mathrm{~h}$ & $23.6 \mathrm{~h}$ & $N R$ & $N R$ & $N R$ & $N R$ & $N R$ & $100 \%$ & $N R$ & $N R$ & NR & \\
\hline & & & $\mathrm{AS}+\mathrm{PQ}(7+14)$ & 157 & $38.8 \mathrm{~h}$ & $21.6 \mathrm{~h}$ & NR & $N R$ & NR & $N R$ & NR & $100 \%$ & NR & NR & NR & \\
\hline
\end{tabular}


Table 1 Clinical trials of varying design that specifically report on the effectiveness of an artemisinin derivate combined with a blood schizonticide for the treatment of Plasmodium vivax, P. malariae, P. ovale or P. knowlesi malaria (Continued)

\begin{tabular}{|c|c|c|c|c|c|c|c|c|c|c|c|c|c|c|c|c|}
\hline \multirow[t]{8}{*}{$\begin{array}{l}\text { Da Silva Rdo } \\
\text { et al. } 2003[57]^{\beta}\end{array}$} & Brazil & CCT & $\begin{array}{c}\text { (a) } \mathrm{AS}+\mathrm{PQ} \\
(1+7)\end{array}$ & 30 & $N R$ & $N R$ & NR & $96.7 \%$ & $N R$ & $N R$ & $N R$ & $N R$ & NR & NR & NR & $\begin{array}{c}\text { PCT: AS faster than } \\
\text { CQ }(p<0.01)\end{array}$ \\
\hline & & & $\begin{array}{c}\text { (b) } \mathrm{AS}+\mathrm{PQ} \\
(1+7)\end{array}$ & 30 & $N R$ & $N R$ & NR & $100 \%$ & NR & $N R$ & $N R$ & $N R$ & NR & NR & NR & \\
\hline & & & $\begin{array}{c}\text { (c) } \mathrm{AS}+\mathrm{PQ} \\
(1+7)\end{array}$ & 30 & $N R$ & $N R$ & NR & $100 \%$ & NR & $N R$ & $N R$ & $N R$ & $N R$ & NR & NR & $\begin{array}{c}\text { Cure rate: } 92.3 \% \\
\text { (group } A+B+C+G \text { ) vs. } \\
80.2 \% \text { (group } D+E+ \\
F+H) P=0.0372 .\end{array}$ \\
\hline & & & $\begin{array}{l}\text { (d) } \mathrm{AS}+\mathrm{PQ} \\
(1+5)\end{array}$ & 30 & $N R$ & $N R$ & NR & $100 \%$ & NR & NR & NR & NR & NR & $N R$ & NR & \\
\hline & & & $\begin{array}{l}\text { (e) } \mathrm{AS}+\mathrm{PQ} \\
(1+5)\end{array}$ & 26 & NR & $N R$ & NR & $96.2 \%$ & NR & NR & NR & NR & $N R$ & $N R$ & NR & \\
\hline & & & $\begin{array}{l}\text { (f) } \mathrm{AS}+\mathrm{PQ} \\
(1+5)\end{array}$ & 28 & NR & $N R$ & $N R$ & $100 \%$ & $N R$ & $N R$ & $N R$ & $N R$ & $N R$ & NR & $N R$ & \\
\hline & & & $\begin{array}{l}\text { (g) } \mathrm{CQ}+\mathrm{PQ} \\
(1+7)\end{array}$ & 30 & NR & NR & NR & $76.7 \%$ & NR & NR & NR & NR & NR & NR & NR & \\
\hline & & & (h) $\mathrm{CQ}+\mathrm{PQ}(1+5)$ & 30 & $N R$ & NR & NR & $60.0 \%$ & NR & NR & $N R$ & NR & NR & NR & NR & \\
\hline \multirow{3}{*}{$\begin{array}{l}\text { Tjitra et al. } 2002 \\
{[37]^{a}}\end{array}$} & Papua, Indonesia & ССТ & $\mathrm{AS}+\mathrm{SP}(3+1)$ & 22 & $1.1 d$ & $1.4 d$ & NR & NR & NR & $100 \%$ & $89.5 \%$ & $N R$ & $N R$ & NR & NR & $C Q$ vs. $C Q+S P p=0.046$ \\
\hline & & & $\mathrm{CQ}+\mathrm{SP}(3+1)$ & 6 & NR & NR & NR & NR & NR & NR & $67 \%$ & $N R$ & $N R$ & NR & NR & \\
\hline & & & CQ (3) & 9 & $N R$ & $N R$ & NR & NR & NR & NR & $11 \%$ & $N R$ & $N R$ & NR & NR & \\
\hline \multirow{2}{*}{$\begin{array}{l}\text { Phan et al. } 2002 \\
{[58]^{a}}\end{array}$} & Vietnam & RCT & AM (3) & 113 & $24 \mathrm{~h}$ & $16 \mathrm{~h}$ & NR & NR & NR & NR & $77 \%$ & NR & NR & NR & NR & Day 28: $p=0.3$ \\
\hline & & & CQ (3) & 113 & $24 \mathrm{~h}$ & $16 \mathrm{~h}$ & NR & NR & NR & NR & $83 \%$ & NR & NR & NR & NR & \\
\hline \multirow{9}{*}{$\begin{array}{l}\text { Pukrittayakamee } \\
\text { et al. } 2000 \text { [59] }\end{array}$} & Thailand & RCT & AS (5) & 20 & $38 \mathrm{~h}$ & $14 \mathrm{~h}$ & NR & NR & $N R$ & NR & $36.8 \%$ & NR & $N R$ & NR & NR & PCT: \\
\hline & & & $\mathrm{AH}(5)$ & 20 & $50 \mathrm{~h}$ & $17 \mathrm{~h}$ & NR & NR & NR & NR & $47.0 \%$ & NR & NR & NR & NR & $C Q+P Q$ vs. $A S p<0.005$ \\
\hline & & & PQ (14) & 30 & $93 \mathrm{~h}$ & $28 \mathrm{~h}$ & $N R$ & $N R$ & NR & NR & $76.9 \%$ & $N R$ & $N R$ & $N R$ & $N R$ & $\begin{array}{c}\mathrm{CQ}+\mathrm{PQ} \text { vs. } \mathrm{QN}, \mathrm{PQ} \\
\text { and } \mathrm{SP} p<0.001\end{array}$ \\
\hline & & & $\mathrm{SP}(1)$ & 12 & $114 \mathrm{~h}$ & $58 \mathrm{~h}$ & NR & NR & NR & $N R$ & $20 \%$ & NR & NR & $N R$ & NR & $\begin{array}{c}\mathrm{CQ}+\mathrm{PQ} \text { and } \mathrm{MQ} \text { vs. } \\
\text { others } \mathrm{P}<0.017\end{array}$ \\
\hline & & & CQ (3) & 30 & $65 \mathrm{~h}$ & $31 \mathrm{~h}$ & NR & $N R$ & $N R$ & NR & $71.4 \%$ & $N R$ & NR & $N R$ & NR & \\
\hline & & & QN (7) & 22 & $98 \mathrm{~h}$ & $31 \mathrm{~h}$ & NR & $N R$ & $N R$ & NR & $35.3 \%$ & $N R$ & NR & $N R$ & $N R$ & \\
\hline & & & MQ (1) & 20 & $76 \mathrm{~h}$ & $21 \mathrm{~h}$ & NR & $N R$ & NR & NR & $100 \%$ & $N R$ & NR & $N R$ & $N R$ & \\
\hline & & & $\mathrm{HT}(1)$ & 23 & $85 \mathrm{~h}$ & $35 \mathrm{~h}$ & NR & NR & NR & NR & $35.3 \%$ & $N R$ & NR & NR & NR & \\
\hline & & & $C Q+P Q(3+14)$ & 30 & $65 \mathrm{~h}$ & $30 \mathrm{~h}$ & NR & $N R$ & $N R$ & NR & $100 \%$ & $N R$ & NR & $N R$ & $N R$ & \\
\hline
\end{tabular}


Table 1 Clinical trials of varying design that specifically report on the effectiveness of an artemisinin derivate combined with a blood schizonticide for the treatment of Plasmodium vivax, P. malariae, P. ovale or P. knowlesi malaria (Continued)

\begin{tabular}{|c|c|c|c|c|c|c|c|c|c|c|c|c|c|c|c|c|}
\hline \multirow[t]{4}{*}{$\begin{array}{l}\text { Li et al., } 1999 \\
\text { [30] NR }\end{array}$} & China & NR & $\begin{array}{c}\mathrm{AL}(3) \\
\text { (higher dose) }\end{array}$ & 36 & $33.5 \mathrm{~h}$ & $22.3 \mathrm{~h}$ & NR & $N R$ & NR & $N R$ & NR & NR & $N R$ & NR & NR & $\mathrm{PCT}: \mathrm{p}<0.01$ \\
\hline & & & & & & & & & & & & & & & & $F C T: p>0.05$ \\
\hline & & & $\begin{array}{c}\text { AL (3) } \\
\text { (lower dose) }\end{array}$ & 41 & $30.5 \mathrm{~h}$ & $22.3 \mathrm{~h}$ & NR & NR & NR & NR & NR & NR & NR & NR & NR & $\begin{array}{l}\text { Relapse rates Month 9: } \\
84.9 \%, 78.8 \% \text { and } 22.9 \%\end{array}$ \\
\hline & & & $\mathrm{CQ}+\mathrm{PQ}(\mathrm{NR})$ & 55 & $44.9 \mathrm{~h}$ & $25.0 \mathrm{~h}$ & $N R$ & $N R$ & $N R$ & $N R$ & NR & $N R$ & $N R$ & $N R$ & NR & \\
\hline \multirow[t]{4}{*}{$\begin{array}{l}\text { Wilairatana et al., } \\
1999 \text { [60] }\end{array}$} & Thailand & CCT & $\mathrm{SP}(1)$ & 23 & $123.1 \mathrm{~h}$ & $49.5 \mathrm{~h}$ & NR & NR & NR & NR & NR & $40 \%$ & NR & NR & NR & $\begin{array}{c}A S+P Q \text { vs. others } \\
P<0.001\end{array}$ \\
\hline & & & $S P+P Q(1+14)$ & 23 & $96.8 \mathrm{~h}$ & $43.2 \mathrm{~h}$ & NR & NR & NR & NR & NR & $100 \%$ & NR & NR & NR & \\
\hline & & & $\mathrm{PQ}(14)$ & 23 & $85.1 \mathrm{~h}$ & $37.7 \mathrm{~h}$ & NR & NR & NR & NR & NR & $100 \%$ & NR & NR & NR & \\
\hline & & & $\mathrm{AS}+\mathrm{PQ}(3+14)$ & 23 & $41.1 \mathrm{~h}$ & $16.4 \mathrm{~h}$ & NR & NR & NR & NR & NR & $100 \%$ & NR & NR & NR & \\
\hline \multicolumn{17}{|l|}{ P. ovale } \\
\hline $\begin{array}{l}\text { Same-Ekobo et al., } \\
1999 \text { [61] NR }\end{array}$ & Cameroon & $\mathrm{CT}$ & AS (NR) & 30 & $38.8 \mathrm{~h}$ & $36.6 \mathrm{~h}$ & NR & $N R$ & NR & $N R$ & NR & NR & NR & NR & NR & \\
\hline \multicolumn{17}{|c|}{ Plasmodium malariae } \\
\hline \multirow{2}{*}{$\begin{array}{l}\text { Borrmann et al. } \\
2002[62]^{a}\end{array}$} & Gabon & $\mathrm{CCT}$ & AS (3) & 35 & NR & NR & NR & NR & NR & $100 \%$ & NR & NR & NR & $83 \%$ & NR & Day 7: $p<0.0001$ \\
\hline & & & Placebo (3) & 23 & NR & NR & NR & NR & NR & $0 \%$ & NR & NR & NR & $0 \%$ & NR & Day 56: $p<0.0001$ \\
\hline \multicolumn{17}{|c|}{ Mixed, Plasmodium ovale and/or Plasmodium malariae and/or Plasmodium falciparum } \\
\hline $\begin{array}{l}\text { Mombo-ngoma } \\
\text { et al. } 2012[8]^{a}\end{array}$ & Gabon & $\mathrm{CT}$ & $\mathrm{AL}(3)$ & 38 & $24 \mathrm{~h}$ & $N R$ & $82 \%$ & $100 \%$ & NR & NR & NR & $100 \%$ & NR & NR & NR & \\
\hline \multicolumn{17}{|c|}{ Mixed infections: Plasmodium falciparum and Plasmodium vivax } \\
\hline \multirow[t]{3}{*}{$\begin{array}{l}\text { Tititra et al. } 2012 \\
{[63]^{a}}\end{array}$} & Indonesia & $\mathrm{RCT}$ & AN (1) & 401 & $13.3 \mathrm{~h}$ & $28.0 \mathrm{~h}$ & NR & NR & NR & NR & NR & NR & $96.3 \%$ & NR & NR & $\begin{array}{c}153(38 \%) \text { P.f., } 158 \text { (39\%) } \\
\text { P.v., } 90(22 \%) \text { P.f./P.v. }\end{array}$ \\
\hline & & & DHP (3) & & $11.3 \mathrm{~h}$ & $25.5 \mathrm{~h}$ & & & & & & & $97.3 \%$ & & & \\
\hline & & & & & & & & & & & & & (AM) & & & \\
\hline \multirow[t]{5}{*}{$\begin{array}{l}\text { Smithuis et al. } \\
2010 \text { [9] }\end{array}$} & Myanmar (Burma) & $\begin{array}{l}\text { RCT, open } \\
\text { label }\end{array}$ & AAQ & 155 & NR & NR & NR & NR & NR & NR & NR & $92 \%$ & NR & NR & $90.6 \%$ & $\begin{array}{c}129(16 \%) \text { mixed } \\
\text { infections P.f. and P.V. }\end{array}$ \\
\hline & & & $\mathrm{AL}$ & 162 & & & & & & & & $99.4 \%$ & & & $98.6 \%$ & \\
\hline & & & AS-MQ (fixed) & 169 & & & & & & & & $100 \%$ & & & $100 \%$ & \\
\hline & & & AS-MQ (loose) & 161 & & & & & & & & $99.4 \%$ & & & $98.7 \%$ & \\
\hline & & & DHP & 161 & & & & & & & & $100 \%$ & & & $98.7 \%$ & \\
\hline
\end{tabular}


Table 1 Clinical trials of varying design that specifically report on the effectiveness of an artemisinin derivate combined with a blood schizonticide for the treatment of Plasmodium vivax, P. malariae, P. ovale or P. knowlesi malaria (Continued)

\begin{tabular}{|c|c|c|c|c|c|c|c|c|c|c|c|c|c|c|c|c|}
\hline \multirow[t]{3}{*}{$\begin{array}{l}\text { Asley et al. } \\
2005 \text { [64] }\end{array}$} & Thailand & RCT & DHP (3) & 164 & $<48 \mathrm{~h}$ & $<48 \mathrm{~h}$ & NR & NR & NR & NR & NR & NR & NR & NR & $100 \%$ & $\begin{array}{c}45 \text { of } 499 \text { patients (9\%) } \\
\text { with P.V. > Results for } \\
\text { any malaria. }\end{array}$ \\
\hline & & & $\mathrm{DHP}(1)$ & 169 & $<48 \mathrm{~h}$ & $<48 \mathrm{~h}$ & & & & & & & & & $99.4 \%$ & \\
\hline & & & MQ-AS & 166 & $<48 \mathrm{~h}$ & $<48 \mathrm{~h}$ & & & & & & & & & $95.7 \%$ & \\
\hline \multicolumn{17}{|c|}{ Plasmodium knowlesi } \\
\hline \multirow[t]{3}{*}{$\begin{array}{l}\text { Barber et al. } \\
2011 \text { [65] }\end{array}$} & Malaysian Borneo & CT & $\mathrm{AL}$ & 109 & $48 \mathrm{~h}$ & $24 \mathrm{~h}$ & NR & NR & NR & NR & NR & NR & $\begin{array}{l}1 \text { patient } \\
\text { with } \\
\text { recurrent } \\
\text { P.k. day } 42\end{array}$ & NR & NR & $\begin{array}{c}\text { Total } 130 \text { P.k. patients. } 23 \\
\text { (18\%) of P.k. patient with } \\
\text { follow up } 26-41 \text { days, no } \\
\text { recurrences. }\end{array}$ \\
\hline & & & AS-MQ & 10 & & & & & & & & & & & & \\
\hline & & & (i.v. AS for SM) & 36 & & & & & & & & & & & & \\
\hline \multirow{2}{*}{$\begin{array}{l}\text { William et al. } \\
2011 \text { [66] }\end{array}$} & Malaysian Borneo & CT & $A L$ & 8 & $24 \mathrm{~h}$ & NR & NR & $N R$ & NR & NR & NR & NR & $N R$ & NR & NR & NR \\
\hline & & & AS (for SM) & 6 & $48 \mathrm{~h}$ & & & & & & & & & & & \\
\hline
\end{tabular}

Case-series and case-reports are not depicted.

AS (for SM) $\quad 6 \quad 48 \mathrm{~h}$

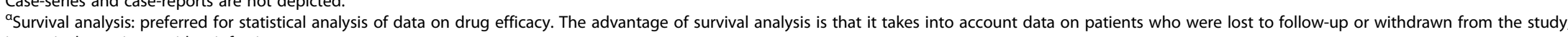
in particular patients with reinfection.

${ }^{\beta}$ Per-protocol analysis: in this method, all patients who cannot be evaluated (i.e. those withdrawn, lost to follow-up or reinfected after PCR correction) are removed from the denominator. 
for severe $P$. vivax malaria [42]. ACT clears $P$. vivax parasitaemia significantly faster than CQ [43] (Table 1). In addition, $\mathrm{ACT}$ also appears to reduce fever clearance time faster than CQ (Table 1). Overall, in regions where there is no CQ resistant $P$. vivax malaria, ACT is as effective as CQ at preventing recurrent parasitaemia up to day $28[31,34-36,39,44]$. Five trials $[28,34-36,44]$ followed up beyond day 28 , but in only one, primaquine was administered to achieve radical cure [39]. It was demonstrated that AS + PY followed by PQ reduced the risk of recrudescent parasitaemia between day 28 and 42 [39]. The longest follow-up was 63 days in a study testing DHA + PP, where DHA + PP treated patients had a $79.1 \% \mathrm{cu}-$ mulative risk of recurrence with $P$. vivax at nine weeks (95\% CI 73.5\%-84.8\%) in patients treated with CQ, compared with $54.9 \%$ (95\% CI, 48.2\%-61.6\%) in those receiving DHA + PP [34]. In all trials comparing ACT with $\mathrm{CQ}$, serious adverse events were rare. ACT has also been investigated in non-randomized clinical trials with $\mathrm{AL}$ [32,45-47] and injectable artesunate (for severe P. vivax malaria) $[25,26,41,42,48,49]$, all showing relative short parasite and fever clearance times and excellent day 28 cure rates.

\section{$\mathrm{AS}+\mathrm{SP}$}

Only one artemisinin combination, namely AS + SP, seems unsuitable for the treatment of vivax malaria. Although one non-inferiority trial [36], from Afghanistan showed good efficacy (day 28 cure rate AS + SP 99\% vs CQ 96\%), it seems that $P$. vivax has developed resistance to pyrimethamine faster than P. falciparum did [67]. Consequently, AS + SP may not be effective overall against $P$. vivax in many regions.

\section{$\mathrm{ACT}$ versus $\mathrm{CQ}+\mathrm{SP}$}

Two trials investigated an $A C T$ versus $C Q+S P$; one study compared AS + SP with CQ + SP [37], and one trial compared three artemisinin-based combinations (DHA + PP, AL and AS + SP) with CQ + SP [38]. High recurrence rates were noted, due to high levels of $\mathrm{CQ}$ and/or SP resistance at the study site. The best form of $\mathrm{ACT}$ in this trial was DHA + PP, with a day 28 cure rate of $84.2 \%$, significantly better than $\mathrm{CQ}+\mathrm{SP}$ [38]. Only one trial evaluated the efficacy of ACT compared to quinine [52]. The efficacy of primaquine (PQ) against relapse was $92 \%$ (95\% CI 81\%-96\%) for quinine plus PQ and 98\% (95\% CI 91\%-99\%) for DHA + PP plus PQ.

\section{ACT versus ACT}

Six studies [38,50-52,54,55,64] evaluated different artemisinin-based combinations. Treatment efficacy with $\mathrm{DHA}+\mathrm{PP}$ was superior to AL, with higher cure rates at day $28[38,55]$. DHA + PP was also more effective compared to AS + AQ [54] and AS + SP [38] while there was no difference to AS + MQ [64] in preventing recurrent parasitaemia. Thus, most evidence is available on the efficacy and safety of DHA + PP for the treatment of $P$. vivax $[28,33,34,38,50-52,54,55,68]$. As depicted in Table 1, no evidence was found on superiority of one regimen over others regarding the parasite and fever clearance time.

An RCT evaluating a two versus three-day regimen of DHA + PP (total dose $360 \mathrm{mg}$ DHA and $2880 \mathrm{mg}$ PP) for uncomplicated malaria ( $75 \%$ P. vivax, $6,3 \%$ mixed) showed no difference in efficacy for treatment of $P$. vivax or all-species malaria before day 42 [50]. Although piperaquine is generally well tolerated with few side effects, there was a safety signal suggested by the degree of QT interval prolongation. The study reported a mean QT prolongation of $20-30 \mathrm{~ms}$ between pre-dose and trough drug piperaquine levels, and roughly $18.5 \%$ of the 80 subjects dosed had a grade I cardiac adverse event due to QT prolongation during the 3 day period following dosing. There were no differences in adverse cardiac events between the two groups. More recently, a randomized, double-blind, placebo-controlled clinical trial of a compressed two-day regimen of DHA + PP for malaria protective efficacy was halted after 6 weeks (69 of 231 participants enrolled) for concern over prolonged corrected QT interval [69]. Participants received two consecutive daily doses (total dose $360 \mathrm{mg}$ DHA and $2880 \mathrm{mg}$ PP) within $30 \mathrm{~min}$ to 3 hour of a meal. The data was reviewed (unblinded) by the data safety monitoring board and they revealed a mean QTcF prolongation of $46 \mathrm{~ms}$ over placebo at the maximum concentration of drug in serum $\left(\mathrm{C}_{\max }\right)$ on day 2 . The authors concluded that a compressed 2-day regimen is best avoided until the clinical relevance of these findings are more thoroughly evaluated.

Evidence from trials evaluating ACT for non-falciparum shows good efficacy of various artemisinin-based combinations for mixed species infection $[9,63]$. In an RCT in Myanmar (Burma) 129 (16\%) of patients had a mixed species infection, and all patients with mixed infections responded to ACT treatment [9]. Fewer cases of $P$. vivax were identified in those who received artesunatemefloquine than in patients who received artesunateamodiaquine, artemether-lumefantrine, or the loose tablet regimen of artesunate-mefloquine [9].

\section{Treatment of ovale and malariae malaria}

Epidemiology of $P$. ovale and $P$. malariae, clinical presentation and diagnosis are described in a recent review [70] and briefly in Additional file 3. Only three trials evaluating an artemisinin derivative for $P$. ovale or $P$. malariae could be identified $[8,61,62]$. A clinical study evaluated the efficacy and tolerability of a three-day 
course of artesunate monotherapy for the clearance of asymptomatic P. malariae infections in Gabon [62]. Patients were randomized and received artesunate $(n=63)$ or placebo $(\mathrm{n}=45)$. Most were mixed infections with $P$. falciparum and 18 were asymptomatic $P$. malariae mono-infections. On day 7 , the cure rate was $100 \%$ (95\% CI 91-100\%) for P. malariae patients treated with artesunate versus no cure (95\% CI 0-15\%) in the placebo group. Treatment with artesunate led to a parasitological cure rate of $83 \%$ by day 56 in this semi-immune population. However, there may have been possible re-infections during the 56-day follow-up period. The true cure rate is likely to be underestimated as these results were not corrected for by PCR. In another clinical trial, artesunate was $100 \%$ effective for the treatment of $P$. ovale infections, with a PCT and FCT of $<48$ hours [61]. Treatment was well tolerated and serious adverse events were not reported. Another study in Gabon, a prospective non-randomized trial evaluated the safety and efficacy of an ACT (AL) for non-falciparum malaria [8]. Most patients in this study had a mixed infection $(n=32)$ and 7 patients presented with a non-falciparum monoinfection. The median parasite clearance time was 36 hours, median FCT 8 hours, and all patients had an adequate clinical and parasitological response at day 28 (95\% CI 91-100\%). No serious adverse events were recorded. The limitations of this study were its nonrandomized design, short follow-up period of 28 days (likely to miss any relapse of $P$. ovale) and no antirelapse therapy with primaquine administered for $P$. ovale. One other case report is available, which reported good efficacy of intravenous artesunate for mixed $P$. falciparum/P. malariae infection [71]. This stands in contrast to a case report from Italy, in which they described a mixed $P$. falciparum $/ P$. malariae infection in a HIV-infected male and ineffectiveness of artemether/lumefantrine to clear the infection of P. malariae [72]. This could be explained by the ineffectiveness of AL against the long-lasting schizogonic pre-erythrocytic phase of $P$. malariae or a natural induced drug resistance of the parasite, which have never been published before in quartan malaria. However, single case reports leave open questions, and hypotheses on lack of ACT efficacy against $P$. malariae remain to be somewhat weak.

\section{Treatment of Plasmodium knowlesi malaria}

A detailed overview on the history, clinical symptoms and diagnosis of $P$. knowlesi malaria is provided in a recent review [73]. Because experience is limited to low case numbers, and as there are no high-quality clinical studies with adequate patient numbers for understandable reasons, current WHO Malaria Treatment Guidelines (2010) [16] do not provide official treatment recommendations yet for uncomplicated knowlesi malaria. For severe knowlesi malaria, WHO recommends intravenous artesunate [74] in their practical handbook 'Management of severe malaria (2013). Taking into account the biology of the parasite and the resulting clinical features with possible rapid progress to severe disease, ACT appear as a natural choice for first-line treatment. Consequently, hospital guidelines in endemic areas currently recommend an oral ACT (AL) for non-severe $P$. knowlesi malaria, and intravenous artesunate for severe knowlesi malaria [46]. A recent study from Barber and colleagues confirmed the safety and efficacy of AL $(\mathrm{n}=109)$ or artesunate-mefloquine $(\mathrm{n}=10)$ for uncomplicated $P$. knowlesi infections in humans [46]. The mean parasite clearance time (not separated for $P$. vivax or P. knowlesi), was two days, with $55 / 119$ (42\%) of P. knowlesi infected patients being smear-negative by day $1(\mathrm{P}=0.032)$. The median fever clearance time was one day in P. knowlesi compared to two days for P. falciparum malaria. Twenty-three (18\%) P. knowlesi patients were followed up during days 26-41, with no recrudescence identified. For severe knowlesi malaria, intravenous artesunate was successfully used. In this study, $>80 \%$ (15 of 18 ) of the patients with a P. knowlesi parasitaemia $>100,000$ parasites $/ \mu \mathrm{L}$ met other severity criteria, supporting the use of this cut-off value for mandating intravenous artesunate. William and colleagues demonstrated excellent efficacy of AL $(n=8)$ for uncomplicated knowlesi malaria in a retrospective case review study [66]. The median parasite clearance time was significantly shorter for patients receiving AL (one day; range 0-3) and for those receiving CQ (2.5 days; range $1-3)$ or quinine (2.5 days; range $1-3)(\mathrm{p}=0.01)$ [66]. Of the 22 patients with severe $P$. knowlesi malaria, 16 were treated with intravenous quinine, six received intravenous artesunate. The median PCT was significantly shorter with artesunate (2 days; range $1-3$ ) than with quinine (4 days; range $2-7)(p=0.02)$. Six patients died, of which 5 had been administered quinine (median severity criteria 2 ; case-fatality rate $31 \%$ ), and 1 was given artesunate (median severity criteria 2.5; case-fatality rate $16.6 \%$ ). Only a few others report on the treatment of P. knowlesi with ACT. A patient from Pahang Province (Malaysia) was unsuccessfully treated with mefloquine, but recovered with intravenous quinine in combination with artemether-lumefantrine [75]. Another case report describes a case of $P$. knowlesi infection in a traveller from Thailand in Germany. Parenteral treatment was started $(2.4 \mathrm{mg} / \mathrm{kg}$ ) and the patient was switched to oral AL the next day, on which she fully recovered [76].

\section{Discussion}

Evidence for the efficacy of ACTs for P. falciparum malaria is extensive, and rapidly growing for $P$. vivax malaria. 
Clinical trials of ACT for P. malariae, $P$. ovale and $P$. knowlesi are much scarcer and limited to non-randomized studies, case-series or case-reports.

\section{Plasmodium vivax malaria}

Several artemisinin-based combinations yield high efficacies against $P$. vivax parasites, both sensitive and resistant to $C Q$. Studies identified in this review showed good efficacy of ACT against the blood stages of $P$. vivax, with quicker parasite and fever clearance times compared to $\mathrm{CQ}$ monotherapy. Countries with CQ-resistant $P$. vivax are already implementing a unified ACT-based treatment policy [77,78]. Increasing resistance of chloroquine against $P$. vivax is, after the first report in 1989 from Papua New Guinea [79], reported in Indonesia [80-83], Myanmar [84], Papua New Guinea [85], Vietnam [86], Turkey [87,88], Colombia [89], Brazil [90], Brazilian Amazon [91] and Ethiopia [29,92-94]. A recent systematic review and meta-analysis reviewed $P$. vivax malaria treatment efficacy studies to establish the global extent of chloroquine resistance [15]. Although there was substantial heterogeneity of study design and analysis between the 129 eligible clinical studies (21,694 patients) chloroquine resistance was present in 58 (53\%) of 113 assessable study sites, spread across most countries that are endemic for $P$. vivax. Also, within countries close to elimination of malaria, an increasing proportion of P. vivax malaria is imported and, outside sub-Saharan Africa, the proportions of all cases caused by $P$. vivax are rising [95]. In travellers returning from supposedly CQ-sensitive $P$. vivax to non-endemic areas, the best treatment remains to be elucidated; in these cases, the economic, malariometric, and operational costs of ACT versus $C Q$ need to be weighted by the clinician. Different regimens are still acceptable if diagnostic tests reliably differentiate CQ-resistant $P$. falciparum from $P$. vivax. However, this is even in experienced laboratories not always the case. Moreover, the paradigm among infectious diseases experts that bacterial infections should be treated as "narrow as possible" does not apply to treating malarial infections. Monotherapy for malaria will only increase the likelihood of resistance, in contrast with narrowing down antibiotic therapy resulting in lower levels of bacterial resistance [96].

A position paper (2012) from the European Society for Clinical Microbiology and Infectious Diseases, summarized main issues regarding the management of imported malaria [97]. For the treatment of non-falciparum malaria, they recommended chloroquine as the drug of choice and $\mathrm{ACT}$ a pragmatic alternative (especially in case of chloroquine resistance). Currently, one trial is recruiting patients to evaluate the efficacy, safety and tolerability of DHA + PP (3 days) with imported P. vivax malaria (NCT02110784).
So far, the use of ACT for non-falciparum malaria, in particular P. vivax, has been jeopardized by the supposedly higher economic costs [98]. This judgement is based on assumption rather than evidence and ignores the malariometric advantages of ACT, declining costs and practical, organizational advantages of a unified ACTbased regimen. Studies evaluating the cost-effectiveness of ACT-based strategy for non-falciparum malaria should be conducted.

The use of ACT for non-complicated non-falciparum malaria infection is convenient but may imply the risks' of a higher utilization of the combination drug, with two possible drawbacks. Firstly, the availability of good quality drugs may be limited in malaria endemic countries [99]; however, there are important caveats to accurately estimate the prevalence and distribution of poor quality anti-malaria drugs. Secondly, inadequate use of ACT (ACT is more expensive than chloroquine) by the population in resource-challenged settings can possibly lead to resistance to both $P$. falciparum and non-falciparum strains [100].

In contrast to $P$. falciparum malaria, $P$. vivax, and to a lesser extent $P$. ovale, form hypnozoites, which remain dormant in liver cells after an acute infection. Therefore, in P. vivax infections (and also P. ovale) the removal of blood schizonts is not sufficient to clear the infection, as the eradication of hypnozoites ("radical cure") is required to prevent relapses. This is clinically important and cost-effective particularly in travellers who are not likely to be re-infected.

ACT does not affect the hypnozoites in the liver, but ACT with a long half-live partner drug, such as piperaquine 23-28 days [101], (compared to CQ 1-2 months [102]) was demonstrated to better prevent recurrent parasitaemia after day $28[34,36,39]$, up to day $42[38,55]$ and 63 [34]. AL which has a short half-life, although less susceptible to the appearance of resistant parasites, is associated with a shorter time to $P$. vivax recurrent parasitaemia. However, neither ACT nor CQ is active against $P$. vivax hypnozoites and thus the treatment must be combined with primaquine, currently the only approved drug able to achieve radical cure. WHO guidelines recommend a course of primaquine for the radical treatment of $P$. vivax of at least 14 days [16]. Although not yet marketed and currently under development, tafenoquine (8-aminoquinoline drug) could lead to a game change in treatment and it may be superior to current treatment due to its radical cure capacity. The proposed indication for tafenoquine is the clearance of the hypnozoite stages of $P$. vivax and $P$. ovale. The assessment of the prophylactic activity and pharmacokinetic profile of oral tafenoquine compared to primaquine for inhibition of liver stage malaria infections demonstrated a much improved efficacy of tafenoquine when 
compared to primaquine [103]. A phase IIb dose-selection study comparing single-dose tafenoquine $300 \mathrm{mg}$ coadministered with CQ for $P$. vivax malaria relapse prevention was more efficacious than $C Q$ alone, with a similar safety profile [104].

\section{Plasmodium ovale and Plasmodium malariae malaria}

At present, $P$. ovale and $P$. malariae are among the most neglected tropical diseases, particularly in sub-Saharan Africa. This is illustrated by the fact that only three clinical trials $[8,61,62]$ were published on ACT for $P$. ovale and P. malariae malaria, compared to a wealth of clinical trials on ACT for P. falciparum malaria [105]. More evidence on the efficacy and safety of ACT in the treatment of $P$. ovale and $P$. malariae is essential before further discouraging the current use of CQ for non-falciparum malaria. Today, the limited evidence available supports both the use of CQ as well as ACT (AL) for $P$. ovale and $P$. malariae. It is advisable to treat with ACT if species classification is not $100 \%$ certain, because $P$. ovale and $P$. malariae often occur in mixed infections with $P$. falciparum, which is likely to be CQ-resistant [8]. Keeping in mind that beyond in vivo testing; in vitro and in silico methods to assess drug resistance of non-falciparum species remain very much limited up to date. $P$. ovale and $P$. malariae are considered sensitive to CQ. However, there is one report on CQ-resistant $P$. malariae from South Sumatra, Indonesia [17]. Simultaneous use of two anti-malarials of different modes of action (as with ACT) will reduce the chance of selection of a resistant strain [96]. Of concern are the results of a trial conducted in Ghanaian schoolchildren, where individuals with microscopically confirmed asymptomatic malaria were treated with dihydroartemisinin-piperaquine and followed for three weeks [106]. A persistent detection of $P$. malariae, $P$. ovale curtisi and $P$. ovale wallikeri was observed. This apparent "treatment failure" requires more investigation. It is important to note, however, that in vivo anti-malarial drug efficacy cannot really be determined from data derived from outcomes of asymptomatic infections. Furthermore, their method of measurement of outcome, PCR, is not yet validated as a primary endpoint method for antimalarial drug trials. It is speculated by the authors that a relapse of $P$. ovale could explain the persistent detection of parasites; however, the prophylactic effect of piperaquine which is generally assumed to last more than three weeks makes this less plausible [106]. Furthermore, it is possible that only gametocytes of $P$. malariae and P. ovale were detected by PCR, and not the asexual stages, as it is known for $P$. falciparum that gametocyte carriage following a dihydroartemisininpiperaquine regimen is more frequent than for other artemisinin-based combinations (e.g. AL) [107]. In $P$. malariae, the long intra-erythrocytic cycle of three days may explain their persistence due to dihydroartemisinin short $\mathrm{T}_{\max }( \pm 2 \mathrm{~h})$ and rapid clearance by CYP3A4/5 in the liver, and, therefore, may be more likely to survive after each dose of artemisinin. Similar observations have been made concerning delayed parasite clearance times in P. malariae in Madagascar, where a patient treated with CQ was still positive for $P$. malariae (detected by PCR) on day 7, although it was subsequently cleared with no late recurrence [108]. Another study, with CQ for $P$. malariae, showed a significant lower clinical response rate, with nearly $20 \%$ of patients still parasitaemic by day 2 and one patient remaining parasitaemic on day 4 [109]. CQ's marked stage specificity of action may account for reports of delayed parasite clearance times [109]. Although highly desirable, randomized controlled trials testing different formulations of ACT versus CQ in the treatment of $P$. ovale and $P$. malariae monoinfections are difficult to realize due to patient's safety issues; misdiagnosis of P. falciparum malaria can have fatal consequences.

\section{Plasmodium knowlesi malaria}

Although currently randomized clinical trials on the treatment of $P$. knowlesi are lacking, several studies have demonstrated the efficacy and safety of ACT (particularly AL) $[65,66]$. Although CQ is efficacious (and cheap) for non-severe P. knowlesi malaria, it is recommended, in view of the potential severity and velocity at which the disease can develop, to use an ACT that is suited for the treatment of $P$. falciparum or $P$. malariae for uncomplicated $P$. knowlesi malaria. This is particularly relevant for elderly patients because they are at greater risk of severe disease due to the strong correlation between age and parasite count $[46,110,111]$. The faster parasite clearance properties of AL are more likely to prevent the most common complication of severe knowlesi malaria; hyperparasitaemia [46]. The importance of early diagnosis [112] and rapid treatment is underlined by the fact that parasite biomass is the major independent risk factor for severe knowlesi malaria [46]. Parenteral artesunate, which is associated with short parasite clearance times, is apparently preferable as treatment of severe knowlesi malaria [46] as it is for falciparum malaria.

In studies conducted in Sarawak, it was demonstrated that whilst most patients with microscopy-diagnosed $P$. malariae infection harboured P. knowlesi; approximately $10 \%$ were actually infected with $P$. falciparum [113]. In travellers returning from Malaysia and other P. knowlesi endemic areas, with a high prevalence of CQ-resistant falciparum malaria, negligent use of CQ for misdiagnosed P. falciparum malaria may have detrimental consequences. Also, resistance of P. knowlesi to anti-malarial drugs have not been observed yet [114]. 
Moreover, artesunate offers a number of advantages over quinine in terms of not requiring rate-controlled infusion or cardiac monitoring [16]. Plasmodium knowlesi was responsible for almost half of the malaria deaths in Sabah (Borneo), due to misdiagnosis with $P$. malariae and delayed administration of artesunate for severe non-falciparum malaria [115]. In a retrospective study, parasite clearance time of AL and injectable artesunate, compared to CQ and quinine, was faster, with fewer deaths with artesunate than quinine [66]. More trials are needed and currently two trials are initiated: "ACT KNOW" (Clinicaltrials.gov: NCT01708876 [116]), a RCT to test whether fixed combination of artesunate-mefloquine is superior to CQ and "CAN KNOW" (NCT02001012), a $\mathrm{RCT}$ to test whether AL is superior to CQ.

\section{Conclusions}

The considerable burden of non-falciparum malaria (predominantly $P$. vivax) in endemic areas and among travellers, the clear recognition as non-benign infection and the emergence of CQ-resistant strains call for a paradigm change in the way non-falciparum malaria infections are treated. Although non-falciparum malaria remains susceptible to $C Q$ in parts of the world, the role of ACT in the treatment of non-falciparum malaria in endemic as well as non-endemic areas should be acknowledged. 'Even' if most non-falciparum infections do not take a potentially life-threatening course, the shorter parasite and fever clearance times alone make a strong case for ACT following establishment of the correct diagnosis. A more uniform treatment for malaria would offer important organizational, logistical and economic advantages, next to malariologic advantages of $\mathrm{ACT}$ in preventing resistance, showing rapid parasite and fever clearance times and good cure rates. However, the incomplete nature of current data on the efficacy of ACT in the treatment of $P$. ovale, $P$. malariae and $P$. knowlesi in endemic areas but also as imported conditions compels current malaria researchers to evaluate the efficacy, safety and cost-effectiveness of these drugs in randomized controlled clinical trials. Because most studies have been carried out in endemic settings, one must be careful transferring these results directly to non-immune travellers with imported non-falciparum malaria. On the other hand, European treatment guidelines are mainly based on data from studies carried out in endemic areas, since there is a paucity of original prospective treatment data for non-immune patients [21]. It must be acknowledged that in most settings, there are too few participants for large-scale trials assessing 'one size fits it all' strategies for non-falciparum malaria in and outside endemic areas. Therefore, the publication of observational data derived from mouse models, pharmacokinetic data from clinical trials should be encouraged. Studies evaluating the cost-effectiveness of ACT-based strategy for non-falciparum malaria should be conducted [117].

\section{Additional files}

Additional file 1: This documents describes the full methods of the present review.

Additional file 2: This PDF file depicts tables with the exact search strategy.

Additional file 3: This document provides basic background information on $P$. ovale, $P$. malariae and $P$. knowlesi infections.

Competing interests

The authors declare that they have no competing interests.

Authors' contributions

BJV, RWW, and MPG conceived the paper. BJV wrote the first draft. DK double-checked the data. IMN conducted the searches and contributed to the methods section. All authors contributed to the concept of the paper and all have been involved in drafting and revising the manuscript. All authors contributed to, and approved the final manuscript.

\section{Acknowledgement}

We thank the reviewer for his/her useful comments on the manuscript.

\section{Author details}

${ }^{1}$ Department of Infectious Diseases, Division of Internal Medicine, Center of Tropical Medicine and Travel Medicine, Academic Medical Center, University of Amsterdam, Meibergdreef 9, PO Box 22700, 1100 DE Amsterdam, The Netherlands. ${ }^{2}$ Centre de Recherches de Médicales de Lambaréné (CERMEL), Albert Schweitzer Hospital, Lambaréné, Gabon. ${ }^{3}$ Institute of Tropical Medicine, University of Tübingen, Tübingen, Germany. "Medical Library, Academic Medical Centre, University of Amsterdam, Amsterdam, The Netherlands. ${ }^{5}$ Department of Paediatric Pneumology and Immunology, Charité-Universitätsmedizin Berlin, Berlin, Germany.

Received: 29 August 2014 Accepted: 18 November 2014 Published: 26 November 2014

\section{References}

1. Jelinek T, Schulte C, Behrens R, Grobusch MP, Coulaud JP, Bisoffi Z, Matteelli A, Clerinx J, Corachan M, Puente S, Gjørup I, Harms G, Kollaritsch H, Kotlowski A, Björkmann A, Delmont JP, Knobloch J, Nielsen LN, Cuadros J, Hatz C, Beran J, Schmid ML, Schulze M, Lopez-Velez R, Fleischer K, Kapaun A, McWhinney P, Kern P, Atougia J, Fry G, et al: Imported Falciparum malaria in Europe: sentinel surveillance data from the European network on surveillance of imported infectious diseases. Clin Infect Dis 2002, 34:572-576.

2. Gautret P, Schlagenhauf $P$, Gaudart J, Castelli F, Brouqui P, von Sonnenburg F, Loutan L, Parola P: Multicenter EuroTravNet/GeoSentinel study of travel-related infectious diseases in Europe. Emerg Infect Dis 2009, 15:1783-1790.

3. Warne B, Weld LH, Cramer JP, Field VK, Grobusch MP, Caumes E, Jensenius M, Gautret P, Schlagenhauf P, Castelli F, Lalloo DG, Ursing J, Chappuis F, von Sonnenburg F, López-Vélez R, Rapp C, Smith KC, Parola P, Gkrania-Klotsas E, EuroTravNet Network: Travel-related infection in European travelers, EuroTravNet 2011. J Travel Med 2014, 21:248-254.

4. Visser BJ, van Vugt M, Grobusch MP: Malaria: an update on current chemotherapy. Exp Opin Pharmacother 2014, 15(15):2219-2254.

5. van Vugt M, van Beest A, Sicuri E, van Tulder M, Grobusch MP: Malaria treatment and prophylaxis in endemic and nonendemic countries: evidence on strategies and their cost-effectiveness. Future Microbio/ 2011, 6:1485-1500.

6. Obare P, Ogutu B, Adams M, Odera JS, Lilley K, Dosoo D, Adhiambo C, Owusu-Agyei S, Binka F, Wanja E, Johnson J: Misclassification of Plasmodium infections by conventional microscopy and the impact of remedial training 
on the proficiency of laboratory technicians in species identification. Malar J 2013, 12:113.

7. Barber BE, William T, Grigg MJ, Yeo TW, Anstey NM: Limitations of microscopy to differentiate Plasmodium species in a region co-endemic for Plasmodium falciparum, Plasmodium vivax and Plasmodium knowlesi. Malar J 2013, 12:8.

8. Mombo-Ngoma G, Kleine C, Basra A, Wurbel H, Diop DA, Capan M, Adegnika AA, Kurth F, Mordmuller B, Joanny F, Kremsner PG, Ramharter M, Bélard S: Prospective evaluation of artemether-lumefantrine for the treatment of non-falciparum and mixed-species malaria in Gabon. Malar J 2012, 11:120.

9. Smithuis F, Kyaw MK, Phe O, Win T, Aung PP, Oo AP, Naing AL, Nyo MY Myint NZ, Imwong M, Ashley E, Lee SJ, White NJ: Effectiveness of five artemisinin combination regimens with or without primaquine in uncomplicated falciparum malaria: an open-label randomised trial. Lancet Infect Dis 2010, 10:673-681.

10. Kilian AH, Metzger WG, Mutschelknauss EJ, Kabagambe G, Langi P, Korte R, von Sonnenburg F: Reliability of malaria microscopy in epidemiological studies: results of quality control. Trop Med Int Health 2000, 5:3-8.

11. McKenzie FE, Sirichaisinthop J, Miller RS, Gasser RA Jr, Wongsrichanalai C: Dependence of malaria detection and species diagnosis by microscopy on parasite density. Am J Trop Med Hyg 2003, 69:372-376.

12. Grobusch MP, Hanscheid T, Zoller T, Jelinek T, Burchard GD: Rapid immunochromatographic malarial antigen detection unreliable for detecting Plasmodium malariae and Plasmodium ovale. Eur J Clin Microbiol Infect Dis 2002, 21:818-820.

13. Foster D, Cox-Singh J, Mohamad DS, Krishna S, Chin PP, Singh B: Evaluation of three rapid diagnostic tests for the detection of human infections with Plasmodium knowlesi. Malar J 2014, 13:60.

14. Baird JK: Chloroquine resistance in Plasmodium vivax. Antimicrob Agents Chemother 2004, 48:4075-4083.

15. Price RN, von Seidlein L, Valecha N, Nosten F, Baird JK, White NJ: Global extent of chloroquine-resistant Plasmodium vivax: a systematic review and meta-analysis. Lancet Infect Dis 2014, 14:982-991.

16. World Health Organization: Guidelines for the Treatment of Malaria. 2nd edition. Geneva, Switzerland: WHO Press, World Health Organization; 2010.

17. Maguire JD, Sumawinata IW, Masbar S, Laksana B, Prodjodipuro P, Susanti I, Sismadi P, Mahmud N, Bangs MJ, Baird JK: Chloroquine-resistant Plasmodium malariae in south Sumatra, Indonesia. Lancet 2002, 360:58-60.

18. Mars U, Larsson BS: Pheomelanin as a binding site for drugs and chemicals. Pigment Cell Res 1999, 12:266-274.

19. Taylor WR, White NJ: Antimalarial drug toxicity: a review. Drug Saf 2004, 27:25-61.

20. Naing C, Whittaker MA, Nyunt Wai V, Mak JW: Is Plasmodium vivax malaria a severe malaria?: a systematic review and meta-analysis. PLoS Negl Trop Dis 2014, 8:e3071.

21. Bouchaud O, Muhlberger N, Parola P, Calleri G, Matteelli A, Peyerl-Hoffmann G, Mechai F, Gautret P, Clerinx J, Kremsner PG, Jelinek T, Kaiser A, Beltrame A, Schmid ML, Kern P, Probst M, Bartoloni A, Weinke T, Grobusch MP: Therapy of uncomplicated falciparum malaria in Europe: MALTHER - a prospective observational multicentre study. Malar J 2012, 11:8.

22. WHO: Methods for surveillance of antimalarial drug efficacy. Geneva: World Health Organization; 2009. ISBN 9789241597531.

23. Liberati A, Altman DG, Tetzlaff J, Mulrow C, Gotzsche PC, loannidis JP, Clarke M, Devereaux PJ, Kleijnen J, Moher D: The PRISMA statement for reporting systematic reviews and meta-analyses of studies that evaluate health care interventions: explanation and elaboration. PLOS Med 2009, 6:e1000100.

24. Efficacy and Safety of Artemisinin Combination Therapy (ACT) for Nonfalciparum Malaria in Travellers: A Systematic Review. [http://www.crd.york.ac. uk/PROSPERO/display_record.asp?ID=CRD42014009103]

25. PiDan L, Yi L, ChangJi M: Observation on the effect of artesunate and artemether on patients with G6PD deficiency vivax malaria patients. China Trop Med 2009, 9:457-458.

26. Yoda T, Rakue Y, Mizota T, Looaresowan S: Clinical trial of efficacy of artesunate against Plasmodium vivax malaria in a Bangkok Hospital. J Tokyo Med Univ 2005, 63:480-484.

27. Muhlberger N, Jelinek T, Gascon J, Probst M, Zoller T, Schunk M, Beran J, Gjorup I, Behrens RH, Clerinx J, Björkman A, McWhinney P, Matteelli A, Lopez-Velez R, Bisoffi Z, Hellgren U, Puente S, Schmid ML, Myrvang B, Holthoff-Stich ML, Laferl H, Hatz C, Kollaritsch H, Kapaun A, Knobloch J, Iversen J, Kotlowski A, Malvy DJ, Kern P, Fry G, et al: Epidemiology and clinical features of vivax malaria imported to Europe: Sentinel surveillance data from TropNetEurop. Malar J 2004, 3:1-7.

28. Hwang J, Alemayehu BH, Reithinger R, Tekleyohannes SG, Takele T, Birhanu SG, Demeke L, Hoos D, Melaku Z, Kassa M, Jima D, Malone JL, Nettey H, Green M, Poe A, Akinyi S, Udhayakumar V, Kachur SP, Filler S: In vivo efficacy of artemether-lumefantrine and chloroquine against Plasmodium vivax: a randomized open label trial in central Ethiopia. PLoS One 2013, 8:e63433.

29. Yohannes AM, Teklehaimanot A, Bergqvist Y, Ringwald P: Confirmed vivax resistance to chloroquine and effectiveness of artemether-lumefantrine for the treatment of vivax malaria in Ethiopia. Am J Trop Med Hyg 2011, 84:137-140.

30. Li X, Li C, Che L, Liu X, Li Z, Huang D, He W, Dao Q: [Observation on efficacy of artemether compound against vivax malaria] (in Chinese). Chung-Kuo Chi Sheng Chung Hsueh Yu Chi Sheng Chung Ping Tsa Chih Chinese J Parasitol Parasitic Dis 1999, 17:175-177.

31. Krudsood S, Tangpukdee N, Muangnoicharoen S, Thanachartwet $V$, Luplertlop N, Srivilairit S, Wilairatana P, Kano S, Ringwald P, Looareesuwan S: Clinical efficacy of chloroquine versus artemether-lumefantrine for Plasmodium vivax treatment in Thailand. Korean J Parasitol 2007, 45:111-114

32. Eibach D, Ceron N, Krishnalall K, Carter K, Bonnot G, Bienvenu AL, Picot S: Therapeutic efficacy of artemether-lumefantrine for Plasmodium vivax infections in a prospective study in Guyana. Malar J 2012, 11:347.

33. Leang R, Barrette A, Bouth DM, Menard D, Abdur R, Duong S, Ringwald P: Efficacy of dihydroartemisinin-piperaquine for treatment of uncomplicated Plasmodium falciparum and Plasmodium vivax in Cambodia, 2008 to 2010. Antimicrob Agents Chemother 2013, 57:818-826.

34. Phyo AP, Lwin KM, Price RN, Ashley EA, Russell B, Sriprawat K, Lindegardh N, Singhasivanon P, White NJ, Nosten F:

Dihydroartemisinin-piperaquine versus chloroquine in the treatment of Plasmodium vivax malaria in Thailand: a randomized controlled trial. Clin Infect Dis 2011, 53:977-984.

35. Awab GR, Pukrittayakamee S, Imwong M, Dondorp AM, Woodrow CJ, Lee SJ, Day NP, Singhasivanon P, White NJ, Kaker F: Dihydroartemisinin-piperaquine versus chloroquine to treat vivax malaria in Afghanistan: an open randomized, non-inferiority, trial. Malar J 2010, 9:105.

36. Kolaczinski K, Durrani N, Rahim S, Rowland M: Sulfadoxine-pyrimethamine plus artesunate compared with chloroquine for the treatment of vivax malaria in areas co-endemic for Plasmodium falciparum and $P$. vivax: a randomised non-inferiority trial in eastern Afghanistan. Trans $R$ Soc Trop Med Hyg 2007, 101:1081-1087.

37. Tjitra E, Baker J, Suprianto S, Cheng Q, Anstey NM: Therapeutic efficacies of artesunate-sulfadoxine-pyrimethamine and chloroquine-sulfadoxine-pyrimethamine in vivax malaria pilot studies: relationship to Plasmodium vivax dhfr mutations. Antimicrob Agents Chemother 2002, 46:3947-3953.

38. Karunajeewa HA, Mueller I, Senn M, Lin E, Law I, Gomorrai PS, Oa O, Griffin S, Kotab K, Suano P, Tarongka N, Ura A, Lautu D, Page-Sharp M, Wong R, Salman S, Siba P, llett KF, Davis TM: A trial of combination antimalarial therapies in children from Papua New Guinea. N Engl J Med 2008, 359:2545-2557.

39. Poravuth $Y$, Socheat $D$, Rueangweerayut $R$, Uthaisin $C$, Pyae Phyo A, Valecha N, Rao BH, Tjitra E, Purnama A, Borghini-Fuhrer I, Duparc S, Shin CS, Fleckenstein L: Pyronaridine-artesunate versus chloroquine in patients with acute Plasmodium vivax malaria: a randomized, double-blind, non-inferiority trial. PLoS One 2011, 6:e14501.

40. Liu R, Dong HF, Jiang MS: A pharmacokinetic approach to assess artemisinin-naphthoquine combination therapy for uncomplicated pediatric malaria. Expert Rev Clin Pharmacol 2012, 5:521-524.

41. Mohapatra MK, Dash LK, Mohapatra A: Severe vivax malaria: a study on its clinical manifestations, risk factors, outcome and therapeutic efficacy of artesunate. Int J Clin Case Reports 2013, 3:doi:10.5376.

42. Dao NV, Cuong BT, Ngoa ND, Duy DN, Dai B, Thanh NX, Chavchich M, Rieckmann $\mathrm{KH}$, Edstein MD: Vivax malaria: preliminary observations following a shorter course of treatment with artesunate plus primaquine. Trans R Soc Trop Med Hyg 2007, 101:534-539.

43. Gogtay N, Kannan S, Thatte UM, Olliaro PL, Sinclair D: Artemisinin-based combination therapy for treating uncomplicated Plasmodium vivax malaria. [Update of Cochrane Database Syst Rev 
2011, 7:CD008492; PMID: 21735431] Cochrane Database of Systematic Rev 2013, 10:CD008492

44. Liu H, Yang HL, Xu JW, Wang JZ, Nie RH, Li CF: Artemisinin-naphthoquine combination versus chloroquine-primaquine to treat vivax malaria: an open-label randomized and non-inferiority trial in Yunnan Province. China Malar J 2013, 12:409.

45. Senn N, Rarau P, Manong D, Salib M, Siba P, Reeder JC, Rogerson SJ, Genton B, Mueller I: Effectiveness of artemether/lumefantrine for the treatment of uncomplicated Plasmodium vivax and $P$. falciparum malaria in young children in Papua New Guinea. Clin Infect Dis 2013, 56:1413-1420.

46. Barber BE, William T, Grigg MJ, Menon J, Auburn S, Marfurt J, Anstey NM, Yeo TW: A prospective comparative study of knowlesi, falciparum, and vivax malaria in Sabah, Malaysia: high proportion with severe disease from Plasmodium knowlesi and Plasmodium vivax but no mortality with early referral and artesunate therapy. Clin Infect Dis 2013, 56:383-397.

47. Abdallah TM, Ali AA, Bakri M, Gasim GI, Musa IR, Adam I: Efficacy of artemether-lumefantrine as a treatment for uncomplicated Plasmodium vivax malaria in eastern Sudan. Malar J 2012, 11:404.

48. Hamedi Y, Safa O, Zare S, Tan-ariya P, Kojima S, Looareesuwan S: Therapeutic efficacy of artesunate in Plasmodium vivax malaria in Thailand. Southeast Asian J Trop Med Public Health 2004, 35:570-574.

49. Karunajeewa HA, Kemiki A, Alpers MP, Lorry K, Batty KT, llett KF, Davis TM: Safety and therapeutic efficacy of artesunate suppositories for treatment of malaria in children in Papua New Guinea. Pediatr Infect Dis J 2003, 22:251-256.

50. Lon C, Manning JE, Vanachayangkul P, So M, Sea D, Se Y, Gosi P, Lanteri C, Chaorattanakawee S, Sriwichai S, Chann S, Kuntawunginn W, Buathong N, Nou S, Walsh DS, Tyner SD, Juliano JJ, Lin J, Spring M, Bethell D, Kaewkungwal J, Tang D, Chuor CM, Satharath P, Saunders D: Efficacy of two versus three-day regimens of dihydroartemisinin-piperaquine for uncomplicated malaria in military personnel in Northern Cambodia: An open-label randomized trial. PLoS One 2014, 9:e93138.

51. Pasaribu AP, Chokejindachai W, Sirivichayakul C, Tanomsing N, Chavez I, Tjitra E, Pasaribu S, Imwong M, White NJ, Dondorp AM: A randomized comparison of dihydroartemisinin-piperaquine and artesunate-amodiaquine combined with primaquine for radical treatment of vivax malaria in Sumatra, Indonesia. J Infect Dis 2013, 208:1906-1913.

52. Sutanto I, Tjahjono B, Basri H, Taylor WR, Putri FA, Meilia RA, Setiabudy R, Nurleila S, Ekawati LL, Elyazar I, Farrar J, Sudoyo H, Baird JK: Randomized, open-label trial of primaquine against vivax malaria relapse in Indonesia. Antimicrob Agents Chemother 2013, 57:1128-1135.

53. Krudsood S, Tangpukdee N, Wilairatana P, Phophak N, Baird JK, Brittenham GM, Looareesuwan S: High-dose primaquine regimens against relapse of Plasmodium vivax malaria. Am J Trop Med Hyg 2008, 78:736-740.

54. Hasugian AR, Purba HL, Kenangalem E, Wuwung RM, Ebsworth EP, Maristela R, Penttinen PM, Laihad F, Anstey NM, Tjitra E, Price RN:

Dihydroartemisinin-piperaquine versus artesunate-amodiaquine: superior efficacy and posttreatment prophylaxis against multidrug-resistant Plasmodium falciparum and Plasmodium vivax malaria. Clin Infect Dis 2007, 44:1067-1074.

55. Ratcliff A, Siswantoro H, Kenangalem E, Maristela R, Wuwung RM, Laihad F, Ebsworth EP, Anstey NM, Tjitra E, Price RN: Two fixed-dose artemisinin combinations for drug-resistant falciparum and vivax malaria in Papua, Indonesia: an open-label randomised comparison. Lancet 2007, 369:757-765.

56. Silachamroon U, Krudsood S, Treeprasertsuk S, Wilairatana P, Chalearmrult K, Mint HY, Maneekan P, White NJ, Gourdeuk VR, Brittenham GM, Looareesuwan S: Clinical trial of oral artesunate with or without high-dose primaquine for the treatment of vivax malaria in Thailand. Am J Trop Med Hyg 2003, 69:14-18.

57. da Silva RS, Pinto AY, Calvosa VS, De Souza JM: [Short course schemes for vivax malaria treatment](in Portuguese). Rev Soc Bras Med Trop 2003, 36:235-239.

58. Phan GT, de Vries PJ, Tran BQ, Le HQ, Nguyen NV, Nguyen TV, Heisterkamp SH, Kager PA: Artemisinin or chloroquine for blood stage Plasmodium vivax malaria in Vietnam. Trop Med Int Health 2002, 7:858-864.

59. Pukrittayakamee S, Chantra A, Simpson JA, Vanijanonta S, Clemens R, Looareesuwan S, White NJ: Therapeutic responses to different antimalarial drugs in vivax malaria. Antimicrob Agents Chemother 2000, 44:1680-1685
60. Wilairatana $P$, Silachamroon $U$, Krudsood $S$, Singhasivanon $P$, Treeprasertsuk S, Bussaratid V, Phumratanaprapin W, Srivilirit S, Looareesuwan S: Efficacy of primaquine regimens for primaquine-resistant Plasmodium vivax malaria in Thailand. Am J Trop Med Hyg 1999, 61:973-977.

61. Same-Ekobo A, Lohoue J, Essono E, Ravinet L, Ducret J-P: [Résolution rapide des acces palustres a Plasmodium ovale par l'artesunate (Arsumax ${ }^{\circledR}$ )](in French). Méd Trop (Mars) 1999, 59:43-45.

62. Borrmann S, Szlezak N, Binder RK, Missinou MA, Lell B, Kremsner PG: Evidence for the efficacy of artesunate in asymptomatic Plasmodium malariae infections. J Antimicrob Chemother 2002, 50:751-754.

63. Tjitra $E$, Hasugian AR, Siswantoro $H$, Prasetyorini B, Ekowatiningsih $R$, Yusnita EA, Purnamasari T, Driyah S, Salwati E, Nurhayati Yuwarni E, Januar L, Labora J, Wijayanto B, Amansyah F, Dedang TA, Purnama A: Trihono: Efficacy and safety of artemisinin-naphthoquine versus dihydroartemisinin-piperaquine in adult patients with uncomplicated malaria: a multi-centre study in Indonesia. Malar J 2012, 11:153.

64. Ashley EA, McGready R, Hutagalung R, Phaiphun L, Slight T, Proux S, Thwai KL, Barends M, Looareesuwan S, White NJ, Nosten F: A randomized, controlled study of a simple, once-daily regimen of dihydroartemisinin-piperaquine for the treatment of uncomplicated, multidrug-resistant falciparum malaria. Clin Infect Dis 2005, 41:425-432.

65. Barber BE, William T, Jikal M, Jilip J, Dhararaj P, Menon J, Yeo TW, Anstey NM: Plasmodium knowlesi malaria in children. Emerg Infect Dis 2011 17:814-820.

66. William T, Menon J, Rajahram G, Chan L, Ma G, Donaldson S, Khoo S Frederick C, Jelip J, Anstey NM, Yeo TW: Severe Plasmodium knowlesi malaria in a tertiary care hospital, Sabah, Malaysia. Emerg Infect Dis 2011, 17:1248-1255

67. Alam MT, Bora H, Bharti PK, Saifi MA, Das MK, Dev V, Kumar A, Singh N, Dash AP, Das B, Wajihullah Sharma YD: Similar trends of pyrimethamine resistance-associated mutations in Plasmodium vivax and $P$. falciparum. Antimicrob Agents Chemother 2007, 51:857-863.

68. Awab GR, Pukrittayakamee S, Jamornthanyawat N, Yamin F, Dondorp AM, Day NPJ, White NJ, Woodrow CJ, Imwong M: Prevalence of antifolate resistance mutations in Plasmodium falciparum isolates in Afghanistan. Malar J 2013, 12:19.

69. Manning J, Vanachayangkul P, Lon C, Spring M, So M, Sea D, Se Y, Somethy S, Phann ST, Chann S, Sriwichai S, Buathong N, Kuntawunginn W, Mitprasat M, Siripokasupkul R, Teja-Isavadharm P, Soh E, Timmermans A, Lanteri C, Kaewkungwal J, Auayporn M, Tang D, Chour CM, Prom S, Haigney M, Cantilena L, Saunders D: Randomized, double-blind, placebo-controlled clinical trial of a two-day regimen of dihydroartemisinin-piperaquine for malaria prevention halted for concern over prolonged corrected QT interval. Antimicrob Agents Chemother 2014, 58(10):6056-6067.

70. Maguire JD, Baird JK: The 'non-falciparum' malarias: the roles of epidemiology, parasite biology, clinical syndromes, complications and diagnostic rigour in guiding therapeutic strategies. Ann Trop Med Parasitol 2010, 104:283-301.

71. Nakajima Y, Suganuma K, Sakamoto M, Osone Y, Akizuki S: [A case of non-Japanese patient with severe co-infection with Plasmodium falciparum and Plasmodium malariae successfully treated with intravenous artesunate](in Japanese). Japanese J Chemother 2009, 57:311-315.

72. Calleri G, Balbiano R, Caramello P: Are artemisinin-based combination therapies effective against Plasmodium malariae? J Antimicrob Chemother 2013, 68:1447-1448.

73. Singh B, Daneshvar C: Human infections and detection of Plasmodium knowlesi. Clin Microbiol Rev 2013, 26:165-184.

74. WHO: Management of Severe Malaria - A Practical Handbook. Thirdth edition. Geneva: World Health Organization; 2013.

75. Lau YL, Tan LH, Chin LC, Fong MY, Noraishah MA, Rohela M: Plasmodium knowlesi reinfection in human. Emerg Infect Dis 2011, 17:1314-1315.

76. Orth $\mathrm{H}$, Jensen BO, Holtfreter MC, Kocheril SJ, Mallach S, MacKenzie C, Muller-Stover I, Henrich B, Imwong M, White NJ, Häussinger D, Richter J: Plasmodium knowlesi infection imported to Germany. Euro Surveill 2013, 18:

77. WHO: World Malaria Report 2013. Geneva: World Health Organization; 2013 [http://www.who.int/malaria/publications/world_malaria_report_2013/ report/en/] 
78. Kusriastuti R, Surya A: New treatment policy of malaria as a part of malaria control program in Indonesia. Acta Med Indones 2012, 44:265-269.

79. Rieckmann $\mathrm{KH}$, Davis DR, Hutton DC: Plasmodium vivax resistance to chloroquine? Lancet 1989, 2:1183-1184.

80. Baird JK, Leksana B, Masbar S, Suradi Sutanihardja MA, Fryauff DJ, Subianto B: Whole blood chloroquine concentrations with Plasmodium vivax infection in Irian Jaya, Indonesia. Am J Trop Med Hyg 1997, 56:618-620.

81. Baird JK, Basri H, Subianto B, Fryauff DJ, McElroy PD, Leksana B, Richie TL, Masbar S, Wignall FS, Hoffman SL: Treatment of chloroquine-resistant Plasmodium vivax with chloroquine and primaquine or halofantrine. J Infect Dis 1995, 171:1678-1682.

82. Ratcliff $A$, Siswantoro $H$, Kenangalem $E$, Wuwung $M$, Brockman A, Edstein MD, Laihad F, Ebsworth EP, Anstey NM, Tjitra E, Price RN: Therapeutic response of multidrug-resistant Plasmodium falciparum and $P$. vivax to chloroquine and sulfadoxine-pyrimethamine in southern Papua, Indonesia. Trans R Soc Trop Med Hyg 2007, 101:351-359.

83. Sumawinata IW, Bernadeta Leksana B, Sutamihardja A, Purnomo Subianto B, Sekartuti Fryauff DJ, Baird JK: Very high risk of therapeutic failure with chloroquine for uncomplicated Plasmodium falciparum and P. vivax malaria in Indonesian Papua. Am J Trop Med Hyg 2003, 68:416-420.

84. Guthmann JP, Pittet A, Lesage A, Imwong $M$, Lindegardh N, Min Lwin M, Zaw T, Annerberg A, de Radigues X, Nosten F: Plasmodium vivax resistance to chloroquine in Dawei, southern Myanmar. Trop Med Int Health 2008, 13:91-98.

85. Genton B, Baea K, Lorry K, Ginny M, Wines B, Alpers MP: Parasitological and clinical efficacy of standard treatment regimens against Plasmodium falciparum, P. vivax and P. malariae in Papua New Guinea. P N G Med J 2005, 48:141-150.

86. Taylor WR, Doan HN, Nguyen DT, Tran TU, Fryauff DJ, Gomez-Saladin E, Kain KC, Le DC, Baird JK: Assessing drug sensitivity of Plasmodium vivax to halofantrine or choroquine in southern, central Vietnam using an extended 28-day in vivo test and polymerase chain reaction genotyping. Am J Trop Med Hyg 2000, 62:693-697.

87. Kurcer MA, Simsek $Z$, Kurcer $Z$ : The decreasing efficacy of chloroquine in the treatment of Plasmodium vivax malaria, in Sanliurfa, south-eastern Turkey. Ann Trop Med Parasitol 2006, 100:109-113.

88. Kurcer MA, Simsek Z, Zeyrek FY, Atay S, Celik H, Kat I, Topluoglu S: Efficacy of chloroquine in the treatment of Plasmodium vivax malaria in Turkey. Ann Trop Med Parasitol 2004, 98:447-451.

89. Soto J, Toledo J, Gutierrez P, Luzz M, Llinas N, Cedeno N, Dunne M, Berman J: Plasmodium vivax clinically resistant to chloroquine in Colombia. Am J Trop Med Hyg 2001, 65:90-93.

90. de Santana Filho FS, Arcanjo AR, Chehuan YM, Costa MR, Martinez-Espinosa FE, Vieira JL, Barbosa M, Alecrim WD, Alecrim M: Chloroquine-resistant Plasmodium vivax, Brazilian Amazon. Emerg Infect Dis 2007, 13:1125-1126

91. Marques MM, Costa MR, Santana Filho FS, Vieira JL, Nascimento MT, Brasil LW, Nogueira F, Silveira H, Reyes-Lecca RC, Monteiro WM, Lacerda MV, Alecrim MG: Plasmodium vivax chloroquine resistance and anemia in the western Brazilian Amazon. Antimicrob Agents Chemother 2014, 58:342-347.

92. Ketema T, Bacha K, Birhanu T, Petros B: Chloroquine-resistant Plasmodium vivax malaria in Serbo town, Jimma zone, south-west Ethiopia. Malar J 2009, 8:177.

93. Teka H, Petros B, Yamuah L, Tesfaye G, Elhassan I, Muchohi S, Kokwaro G Aseffa A, Engers $\mathrm{H}$ : Chloroquine-resistant Plasmodium vivax malaria in Debre Zeit. Ethiopia Malar J 2008, 7:220

94. Mekonnen SK, Aseffa A, Berhe N, Teklehaymanot T, Clouse RM, Gebru T, Medhin G, Velavan TP: Return of chloroquine-sensitive Plasmodium falciparum parasites and emergence of chloroquine-resistant Plasmodium vivax in Ethiopia. Malar J 2014, 13:244.

95. Cotter C, Sturrock HJW, Hsiang MS, Liu J, Phillips AA, Hwang J, Gueye CS, Fullman N, Gosling RD, Feachem RGA: The changing epidemiology of malaria elimination: New strategies for new challenges. Lancet 2013, 382:900-911.

96. White NJ: Delaying antimalarial drug resistance with combination chemotherapy. Parassitologia 1999, 41:301-308.

97. Askling HH, Bruneel F, Burchard G, Castelli F, Chiodini PL, Grobusch MP, Lopez-Velez R, Paul M, Petersen E, Popescu C, Ramharter M, Schlagenhauf P:
European Society for Clinical Microbiology and Infectious Diseases Study Group on Clinical Parasitology: Management of imported malaria in Europe. Malar J 2012, 11:328

98. Whitty CJ, Chandler C, Ansah E, Leslie T, Staedke SG: Deployment of ACT antimalarials for treatment of malaria: challenges and opportunities. Malar J 2008, 7(Suppl 1):S7.

99. Tabernero P, Fernandez FM, Green M, Guerin PJ, Newton PN: Mind the gaps-the epidemiology of poor-quality anti-malarials in the malarious world-analysis of the WorldWide Antimalarial Resistance Network database. Malar J 2014, 13:139.

100. Amaratunga C, Sreng S, Mao S, Tullo GS, Anderson JM, Chuor CM, Suon S, Fairhurst RM: Chloroquine remains effective for treating Plasmodium vivax malaria in Pursat province, Western Cambodia. Antimicrob Agents Chemother 2014, 58:6270-6272.

101. Tarning J, Ashley EA, Lindegardh N, Stepniewska K, Phaiphun L, Day NPJ, McGready R, Ashton M, Nosten F, White NJ: Population pharmacokinetics of piperaquine after two different treatment regimens with dihydroartemisinffi-piperaquine in patients with Plasmodium falciparum malaria in Thailand. Antimicrob Agents Chemother 2008, 52:1052-1061.

102. Krishna S, White NJ: Pharmacokinetics of quinine, chloroquine and amodiaquine. Clinical implications. Clin Pharmacokinet 1996, 30:263-299.

103. Li Q, O'Neil M, Xie L, Caridha D, Zeng Q, Zhang J, Pybus B, Hickman M, Melendez V: Assessment of the prophylactic activity and pharmacokinetic profile of oral tafenoquine compared to primaquine for inhibition of liver stage malaria infections. Malar J 2014, 13:141.

104. Llanos-Cuentas A, Lacerda MV, Rueangweerayut R, Krudsood S, Gupta SK, Kochar SK, Arthur P, Chuenchom N, Mohrle JJ, Duparc S, Ugwuegbulam C, Kleim JP, Carter N, Green JA, Kellam L: Tafenoquine plus chloroquine for the treatment and relapse prevention of Plasmodium vivax malaria (DETECTIVE): a multicentre, double-blind, randomised, phase $2 \mathrm{~b}$ dose-selection study. Lancet 2014, 383:1049-1058.

105. PubMed United States National Library of Medicine, 8600 Rockville Pike, Bethesda, MD 20894. Accessed on December 3 2014. 2001-2014. Available at: www.pubmed.gov.

106. Dinko B, Oguike MC, Larbi JA, Bousema T, Sutherland CJ: Persistent detection of Plasmodium falciparum, $P$. malariae, $P$. ovale curtisi and $P$. ovale wallikeri after ACT treatment of asymptomatic Ghanaian schoolchildren. Int J Parasitol Drugs Drug Resist 2013, 3:45-50.

107. Four Artemisinin-Based Combinations (4ABC) Study Group: A head-to-head comparison of four artemisinin-based combinations for treating uncomplicated malaria in African children: a randomized trial. PLoS Med 2011, 8:e1001119.

108. Barnadas C, Ratsimbasoa A, Ranaivosoa H, Ralaizandry D, Raveloariseheno D, Rabekotonorina V, Picot S, Menard D: Prevalence and chloroquine sensitivity of Plasmodium malariae in Madagascar. Am J Trop Med Hyg 2007, 77:1039-1042.

109. Siswantoro H, Russell B, Ratcliff A, Prasetyorini B, Chalfein F, Marfurt J, Kenangalem E, Wuwung M, Piera KA, Ebsworth EP, Anstey NM, Tjitra E, Price RN: In vivo and in vitro efficacy of chloroquine against Plasmodium malariae and $P$. ovale in Papua, Indonesia. Antimicrob Agents Chemother 2011, 55:197-202.

110. Barber BE: A prospective comparative study of knowlesi, falciparum, and vivax malaria in Sabah, Malaysia: high proportion with severe disease from Plasmodium knowlesi and Plasmodium vivax but no mortality with early referral and artesunate therapy. Clin Infect Dis 2014, 58:608.

111. Muller M, Schlagenhauf P: Plasmodium knowlesi in travellers, update 2013. Int J Infect Dis 2014, 22:55-64.

112. Rajahram GS, Barber BE, Yeo TW, Tan WW, William T: Case report: Fatal Plasmodium knowlesi malaria following an atypical clinical presentation and delayed diagnosis. Med J Malaysia 2013, 68:71-72.

113. Cox-Singh J, Davis TM, Lee KS, Shamsul SS, Matusop A, Ratnam S, Rahman HA, Conway DJ, Singh B: Plasmodium knowlesi malaria in humans is widely distributed and potentially life threatening. Clin Infect Dis 2008, 46:165-171.

114. Kantele A, Jokiranta TS: Review of cases with the emerging fifth human malaria parasite, Plasmodium knowlesi. Clin Infect Dis 2011, 52:1356-1362.

115. Rajahram GS, Barber BE, William T, Menon J, Anstey NM, Yeo TW: Deaths due to Plasmodium knowlesi malaria in Sabah, Malaysia: association with reporting as Plasmodium malariae and delayed parenteral artesunate. Malar J 2012, 11:284 
116. Grigg MJ, William T, Dhanaraj P, Menon J, Barber BE, von Seidlein L, Rajahram G, Price RN, Anstey NM, Yeo TW: A study protocol for a randomised open-label clinical trial of artesunate-mefloquine versus chloroquine in patients with non-severe Plasmodium knowlesi malaria in Sabah, Malaysia (ACT KNOW trial). BMJ Open 2014, 4:e006005.

117. Visser BJ, Buijink AW, Grobusch MP: Reporting of medical research costs. Improving transparency and reproducibility of medical research. Methods Inf Med 2014, 53:329-331.

doi:10.1186/1475-2875-13-463

Cite this article as: Visser et al:: Efficacy and safety of artemisinin combination therapy (ACT) for non-falciparum malaria: a systematic review. Malaria Journal 2014 13:463.

\section{Submit your next manuscript to BioMed Central and take full advantage of:}

- Convenient online submission

- Thorough peer review

- No space constraints or color figure charges

- Immediate publication on acceptance

- Inclusion in PubMed, CAS, Scopus and Google Scholar

- Research which is freely available for redistribution 